\title{
Centipedes (Chilopoda) from the Stavropol Territory, northern Caucasus, Russia
}

\section{Губоногие многоножкки (Chilopoda) Ставропольского края (Северный Кавказ, Россия)}

\author{
R.V. Zuev \\ P.B. Зуев \\ North Caucasus Federal University, Pushkina Str. 1, Stavropol, 355009, Russia. E-mail: romus00@yandex.ru \\ Северо-Кавказский федеральный университет, ул. Пушкина 1, г. Ставрополь, 355009, Россия. \\ KEY WORDS: Chilopoda, faunistics, Stavropol Territory, Ciscaucasia. \\ КЛЮЧЕВЫЕ СЛОВА: Chilopoda, фаунистика, Ставропольский край, Предкавказье.
}

ABSTRACT. The centipede fauna of the Stavropol Territory, northern Caucasus comprises at least 28 species; 15 of them are new to the regional list, including three new to the fauna of the Caucasus: Lithobius melanops Newport, 1845, Schendyla nemorensis (C.L. Koch, 1837) and Diphyonyx conjungens (Verhoeff, 1898). Faunistic records of all these species in the region are presented and mapped.

РЕЗЮМЕ. Фауна губоногих многоножек Ставропольского края включает по крайней мере 28 видов, из них 15 впервые приведены для исследуемой территории, в т.ч. 3 - новые для фауны Кавка3а: Lithobius melanops Newport, 1845, Schendyla nemorensis (C.L. Koch, 1837) и Diphyonyx conjungens (Verhoeff, 1898). Представлены фаунистические находки и карты с указанием распространения этих видов в регионе.

\section{Introduction}

Chilopoda is a class of predatory arthropods which currently contains about 3000 species from five orders. About 80 species from four orders have been reported from Russia.

The centipede fauna of the Stavropol Territory still remains very poorly studied, as the available information relates only to the Caucasian Mineral Waters area and the environs of Stavropol. Sseliwanoff [1881, 1883] recorded seven species of Chilopoda in the region: Lithobius ferganensis (Trotzina, 1894) (originally referred to as L. pusillus Sseliwanoff, 1881), L. elegans Sseliwanoff, 1881, L. mutabilis L. Koch, 1862, L. stuxbergii Sseliwanoff, 1881, Scolopendra cingulata Latreille, 1789, C. hortensis (Donovan, 1810) and Henia bicarinata (Meinert, 1870) (referred to as C. bidenticulatus Sselivanoff, 1884 and Scotophilus bicarinatus Meinert, 1870, respectively). Muralewicz [1907, 1926, 1927, 1929] added another six species: L. colchicus
Muralewicz, 1907, L. liber Lignau, 1903, L. rufus Muralewicz, 1926, L. curtipes C.L. Koch, 1847, Clinopodes caucasicus (Sseliwanoff, 1883) and C. flavidus Koch, 1847 (referred to as Geophilus caucasicus and G. flavi$d u s$, respectively). We consider further six species reported by Sseliwanoff and Muralewicz as erroneous records. Since 1929, there have been no published data on the centipede fauna of the Stavropol Territory.

\section{Material and methods}

The material underlying this paper was collected in 2000-2015 in different places and habitats of the Stavropol Territory. Specimens were taken either by hand or using soil sampling. The material is shared between the collections of the Zoological Museum of the North Caucasian Federal University, Stavropol, Russia (ZMUS) and the Zoological Museum of the State University of Moscow, Russia (ZMUM), as indicated hereafter. Photographs were taken with a DCM 500 digital camera and a Levenhuk DTX 90 digital microscope, images were processed using Adobe Photoshop CS5 software. Altogether, about 1500 specimens have been examined.

\section{Taxonomic part \\ ORDER SCUTIGEROMORPHA \\ Family SCUTIGERIDAE}

Scutigera coleoptrata (Linnaeus, 1758) Map 1.

MATERIAL. $10^{7}$ (ZMUS), Stavropol Territory, Stavropol City, indoors, 10.IV.2012, leg. S.I. Sigida; $1 O^{7}$ (ZMUS), same locality, 30.IX.2011; $1 \sigma^{7}$ (ZMUS), same locality, 22.X.2011; 1 \& (ZMUS), same locality, 25.VI.2012; 1 + (ZMUS), same locality, 26.VII.2012; $1 \mathrm{O}^{\top}$ (ZMUS), same locality, 29.VIII.2014; 1 q (ZMUM), Shpakovsky Distr., near Grushevyi, steppe, 26.X.2013; 1 ○7 (ZMUM), Predgornyi Distr., Energetic, Perkalsky nursery garden, indoors, 


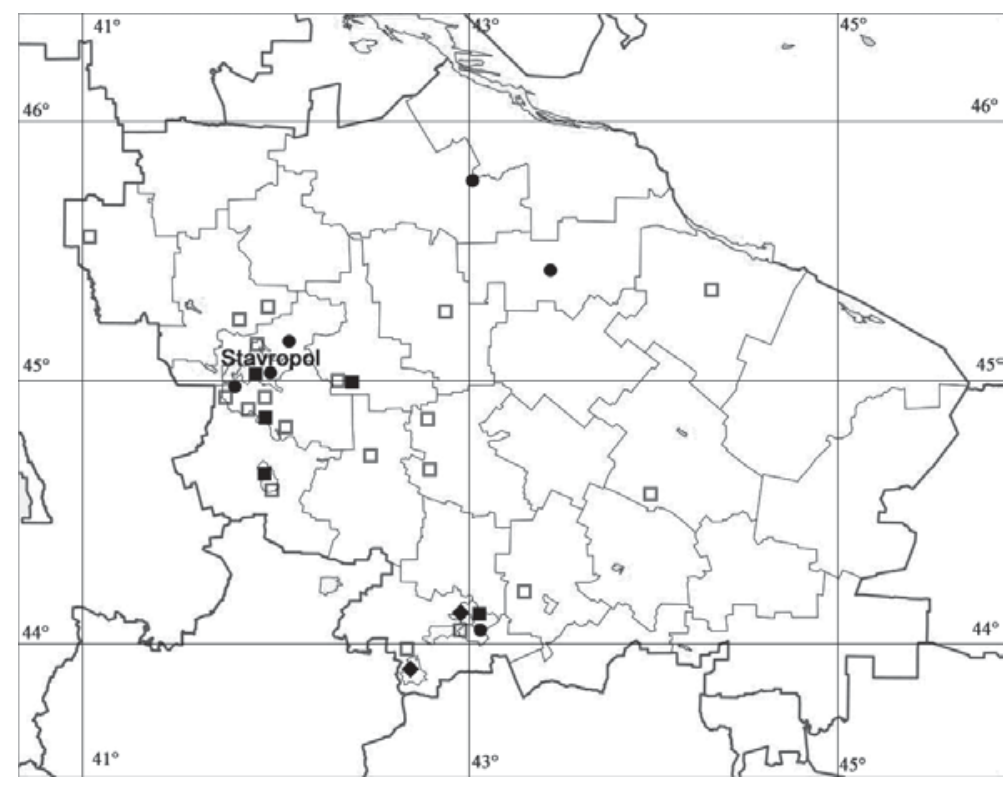

Map 1. Distribution of centipedes in the Stavropol Territory: circle - Scutigera coleoptrata; square - Lithobius colchicus; diamond - L. elegans; open square $-L$. curtipes.

Карта 1. Распространение губоногих многоножек в Ставропольском крае: круг Scutigera coleoptrata; квадрат - Lithobius colchicus; ромб - L. elegans; незакрашенный квадрат - L. curtipes.
21.VII.2013, all leg. R.V. Zuev; 1 juv. (ZMUS), Shpakovsky Distr., Mikhaylovsk, Stavropol Research Institute of Agriculture, forest belt, 4.IV.2008, leg. M.B. Patyuta; $2 O^{\top} O^{\top}, 3$ 3 ㅇ, 2 juv. (ZMUS), Turkmensky Distr., Letnyaya Stavka, 21-28.VI.2014; 1 9 (ZMUS), Ipatovsky Distr., near Liman, 21.VII.2014, all leg. K.S. Priymak.

REMARKS. This Turano-Mediterranean species is widespread throughout Europe, North Africa, West and Central Asia, introduced into South Africa, North and South Americas and Southeast Asia [Simaiakis et al., 2013]. It has been recorded by various authors in Azerbaijan, Georgia and the Black Sea coast of the Caucasus [Eichwald, 1830, 1841; Sseliwanoff, 1883; Lignau, 1903; Muralewicz, 1927, 1929], in the papers by Eichwald and Sseliwanoff being referred to as Scutigera araneoides Pallas, 1772. In the Stavropol Territory, this synanthropous species is most common indoors, being very rare in steppe and forest habitats.

\section{ORDER LITHOBIOMORPHA Family HENICOPIDAE}

Lamyctes (Lamyctes) emarginatus (Newport, 1844)

MATERIAL. 1 \& (ZMUS), Stavropol Territory, Novoaleksandrovsky Distr., near Temigbeksky, field, soil, 25.X.2006, leg. M.B. Patyuta.

REMARKS. A Holarctic species. In Europe, this species is parthenogenetic, being represented by females alone [Zalesskaja, 1978]; in the Caucasus, it has been recorded from Novyi Afon, Abkhazia [Muralewicz, 1926, 1929].

\section{Family LITHOBIIDAE}

Lithobius (Lithobius) colchicus Muralewicz, 1907 Figs 1, 2, Map 1.

MATERIAL. 1 (ZMUM), Stavropol Territory, Stavropol City, Tamansky Forest, 21.III.2013; $2 \sigma^{\top} \sigma^{\top}, 4$ 우 (ZMUM), same locali- ty, 14.IV.2013; 1 ○ (ZMUS), same locality, 15.IV.2013; 1 ㅇ (ZMUS), same locality, 15.VI.2013; 1 (ZMUS), same locality,

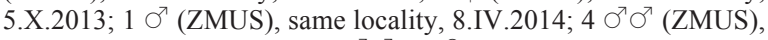
same locality, 1.VI.2014; $2 \sigma^{7} \sigma^{7}, 1$ ( 9 (ZMUS), Russky Forest, 6.VII.2013; 4 O $^{7}$ (ZMUS), same locality, 27.VII.2013; 2 우 (ZMUS), same locality, 2.III.2014; $2 \sigma^{\top} \sigma^{7}$ (ZMUS), same locality, 9.III.2014; $1 \sigma^{7}, 4$ Oᄋ (ZMUS), same locality, 10.V.2014; $3 \sigma^{7} \sigma^{\top}, 6$ of (ZMUS), same locality, 7.VI.2014; 1 (ZMUS), Mamaysky

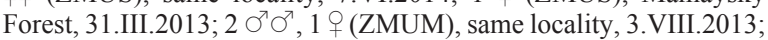
$1 \sigma^{\top}, 3$ 우 (ZMUM), Tatarsky Forest, 5.V.2013; $2 \sigma^{\top} \sigma^{\top}, 2$ 우 (ZMUS), same locality, 19.X.2013; 1 ○ (ZMUS), Shpakovsky Distr., near Rynok, Nedremannaya Mountain, forest, 18.V.2011; 1 $\sigma^{7}$ (ZMUM), Grachevsky Distr., $1 \mathrm{~km} \mathrm{SW}$ of Beshpagir, forest, 17.VII.2014; 1 O' (ZMUM), Kochubeevsky Distr., Nevinnomyssk, confluence of Bolshoy Zelenchuk and Kuban rivers, floodplain forest, 7.IX.2014; 1 O' (ZMUS), Georgievsky Distr., Georgievsk, Safonovsky Forest, 10.VIII.2013; $1 \sigma^{7}$ (ZMUS), Predgornyi Distr., Pyatigorsk, Mashuk Mountain, forest, 20.VII.2013; 1 ㅇ (ZMUM), same locality, 20.VII.2013, all leg. R.V. Zuev.

REMARKS. A Caucasian species which has already been recorded from Stavropol; also it is known from Georgia and the Republic of North Ossetia Alania [Muralewicz, 1907, 1926, 1929].

In the descriptions of this species, the presence of a very small additional claw on the $15^{\text {th }}$ pair of legs was noted. Among the specimens we have found, such an additional claw has only been revealed in a single specimen (Fig. 1), whereas in the remaining material the base of the claw bears only a sensory spine (Fig. 2).

\section{Lithobius (Lithobius) coloratus Sseliwanoff, 1881}

MATERIAL. $1 \overbrace{}^{7}$ (ZMUS), Stavropol Territory, Stavropol City, Russky Forest, 10.V.2014; 1 juv. (ZMUS), Mamaysky Forest, 3.VIII.2013; 1 ๆ (ZMUM), Shpakovsky Distr., Nedremannaya Mountain, forest, 15.VII.2014, all leg. R.V. Zuev.

REMARKS. A Caucasian species known from Georgia, Azerbaijan and the Republic of North Ossetia Alania [Sseliwanoff, 1881; Muralewicz, 1907, 1929; Zalesskaja, 1973]. 


\section{Lithobius (Lithobius) elegans Sseliwanoff, 1881} Fig. 3, Map 1.

MATERIAL. $1 \sigma^{\top}, 1$ (ZMUM), Stavropol Territory, Predgornyi Distr., Pyatigorsk, Mashuk Mountain, forest, 20.VII.2013; 2 $\sigma^{2} \sigma^{2}, 1$ o (ZMUS), Georgievsky Distr., Georgievsk, Safonovsky Forest, 10.VIII.2013, all leg. R.V. Zuev.

REMARKS. A Caucasian species described from near Kislovodsk and Pyatigorsk, Stavropol Territory; in addition, it has been recorded in Georgia, the Republic of North Ossetia - Alania, the Republic of Adygea and the Krasnodar Territory of Russia [Sseliwanoff, 1881; Lignau, 1903, 1914; Muralewicz, 1907, 1929].

Muralewicz described L. striatus striatus, based on material from the Stavropol Territory (Kislovodsk), the Republic of North Ossetia - Alania (Adai-Hoh, Vladikavkaz), the Karachayevo-Cherkessia Republic (Bermamyt), the Republic of Adygea (the upper course of Belaya River) and Georgia (Lagodekhi) [Muralewicz, 1926]. Interestingly, the description, the collecting localities and the number of specimens L. striatus striatus coincided verbatim with a later description of $L$. fasciatus [Muralewicz, 1929; Bonato et al., 2006]. Therefore, Muralewicz seems to have mistakenly described the same species under the names L. striatus striatus and L. fasciatus.

Furthermore, it seems possible to refer L. striatus striatus from Kislovodsk to L. elegans, as the differences between these two species lie in L. striatus striatus showing light stripes on the back and a few peculiarities of female gonopod structure, whereas the single specimen recorded from Kislovodsk by Muralewicz was a male. Muralewicz [1929] emphasized great similarities observed in these species. Although we do not consider them as synonyms, it seems important that there were no additional records of $L$. striatus striatus since Muralewicz's. A female gonopod is illustrated to document the identity (Fig. 3).

\section{Lithobius (Lithobius) forficatus (Linnaeus, 1758) Map 2.}

MATERIAL. 1 \& (ZMUS), Stavropol Territory, Stavropol City, Tamansky Forest, 13.X.2012; 1 juv. (ZMUS), same locality, 5.II.2013; 2 Oొ $^{\top}, 2$ 우 (ZMUS), Mamaysky Forest, 31.III.2013; 1 क (ZMUS), same locality, 3.VIII.2013; 1 O$^{7}, 2$ 우 (ZMUM), Shpakovsky Distr., near Grushevy, steppe, 23.III.2014; 3 juv. (ZMUS), same locality, 23.III.2014; 1 O $^{T}$ (ZMUS), same locality, 14.VI.2014; $10^{7}, 2$ 웅 (ZMUS), same locality, 4.X.2014; 2 juv. (ZMUS), Grachevsky Distr., near Tuguluk, steppe, 21.V.2013; 1 (ZMUS), Alexandrovsky Distr., near Kruglolesskoe, pasture, 26.IV.2014; 1 O (ZMUS), Petrovsky Distr., near Lake Solyonoe, steppe, 10.VII.2014; $2 O^{7} \sigma^{7}, 3$ 우, 1 juv. (ZMUS), Arzgirsky Distr., near Arzgir, steppe, 1.V.2014; 10 $\sigma^{7} \sigma^{7}, 2$ 우 (ZMUM), same locality, 2.V.2014; 1 juv. (ZMUS), Predgornyi Distr., near Kislovodsk, Borgustansky Ridge, steppe, 20.VII.2013, all leg. R.V. Zuev; $1 \mathrm{O}^{7}, 1$ \% (ZMUS), Turkmensky Distr., near Kulikovy Kopani, 24.IV.2011, leg. Romaev; 1 ( (ZMUS), same locality, 25.VI.2014, leg. K.S. Priymak.

REMARKS. A European species recorded in the Caucasus from the Republic of Adygea, the Krasnodar Territory and Georgia [Lignau, 1903, 1915; Attems, 1907; Muralewicz, 1926, 1929; Zalesskaja, 1973]. In
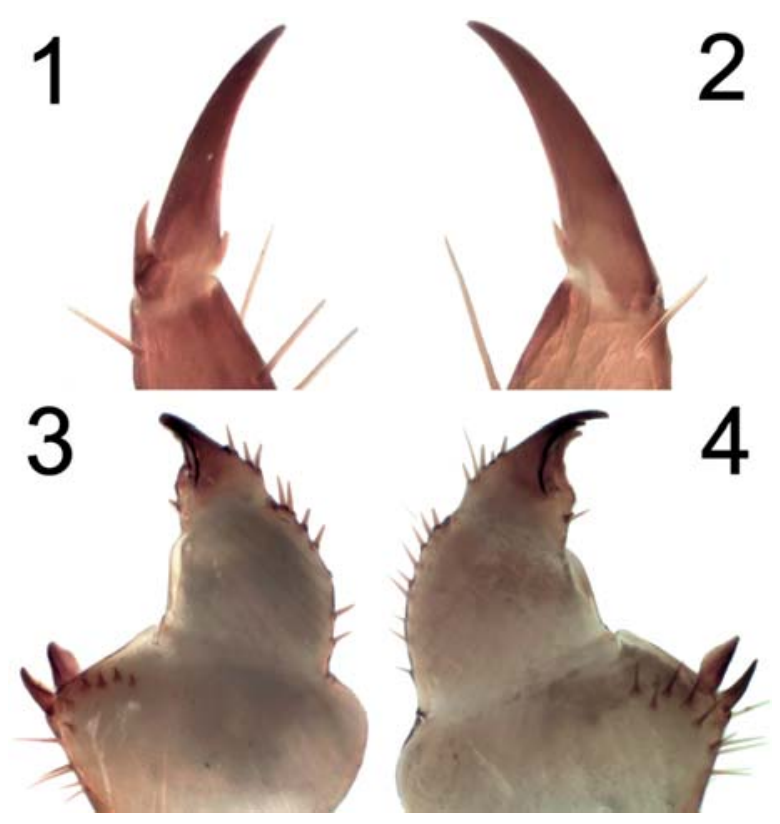

Figs 1-4. Lithobius colchicus Muralewicz, 1907 (1, 2), L. elegans Sseliwanoff, 1881 () (3) and L. liber Lignau, 1903 () (4). 1 - distal part of last left leg, specimen from Beshpagir; 2 distal part of last right leg, specimen from Stavropol; 3 - left gonopod, mesal view; 4 - right gonopod, mesal view. Pictures taken not to scale.

Рис. 1-4. Lithobius colchicus Muralewicz, 1907 (1, 2), L. elegans Sseliwanoff, 1881 (†) (3) и L. liber Lignau, 1903 (†) (4). 1 - дистальная часть конечной левой ноги, экземпляр из Бешпагира; 2 - дистальная часть конечной правой ноги, экземпляр из Ставрополя; 3 - левый гонопод, изнутри; 4 - правый гонопод, изнутри. Фотографии без масштаба.

the Stavropol Territory, it has been found mainly in steppe habitats and anthropogenous landscapes, in the forests being rare.

\section{Lithobius (Lithobius) liber Lignau, 1903} Fig. 4, Map 2.

MATERIAL. $1 \sigma^{\top}, 1$ (ZMUS), Stavropol Territory, Stavropol City, Tamansky Forest, 15.IX.2012; 1 万 (ZMUS), same locality, 5.II.2013; $4 \sigma^{\top} \sigma^{\top}, 4$ 우 (ZMUS), same locality, 21.III.2013; $6 \sigma^{\top} \sigma^{\top}$, 2 우 (ZMUM), same locality, 14.IV.2013; 1 ๙ , 3 우 (ZMUS), same locality, 14.IV.2013; 5 OO (ZMUM), same locality, 28.IV.2013; $1 \sigma^{7}$ (ZMUS), same locality, 15.VI.2013; $2 \sigma^{\top} \sigma^{\top}, 3$ 우 (ZMUS), same locality, 10.IX.2013; 2 ○े $\sigma^{\top}, 2$ 오 (ZMUS), same locality, 12.IX.2013; 1 ○', 1 क (ZMUS), same locality, 5.X.2013; 1 \% (ZMUS), same locality, 9.X.2013; $3 \sigma^{7} \sigma^{\top}, 7$ o+, 1 juv. (ZMUS), same locality, 1.VI.2014; 1 (ZMUS), Mamaysky Forest, 3.VI.2010; 1 ○', 1 ( (ZMUS), same locality, 29.VI.2013; $3 \sigma^{\top} \sigma^{7}$ (ZMUS), same locality, 3.VIII.2013; 1 क (ZMUS), same locality, 16.III.2014;

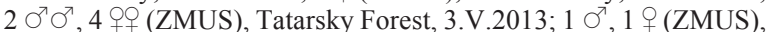
same locality, 19.X.2013; $1 \sigma^{7}, 1$ (ZMUS), Russky Forest, 23.III.2013; 1 య , 1 9 (ZMUM), same locality, 6.IV.2013; 2 우 (ZMUS), same locality, 12.X.2013; $1 \sigma^{7}$ (ZMUS), same locality, 3.XI.2013; 1 ㅇ (ZMUS), same locality, 9.III.2014; 1 ○ (ZMUS), same locality, 10.V.2014; 1 \& (ZMUS), Shpakovsky Distr., 5 km SSE of Izveshchatelnyi, Strizhament Mountain, forest, 21.V.2010; 1 (ZMUS), same locality, 3.VI.2010; 1 \& (ZMUS), same locality, 14.VII.2013; 1 ㅇ (ZMUM), Georgievsky Distr., Georgievsk, Safonovsky Forest, 10.VIII.2013; 1 ऊ (ZMUM), near Alexandriyskaya, forest, 11.VIII.2013, all leg. R.V. Zuev. 


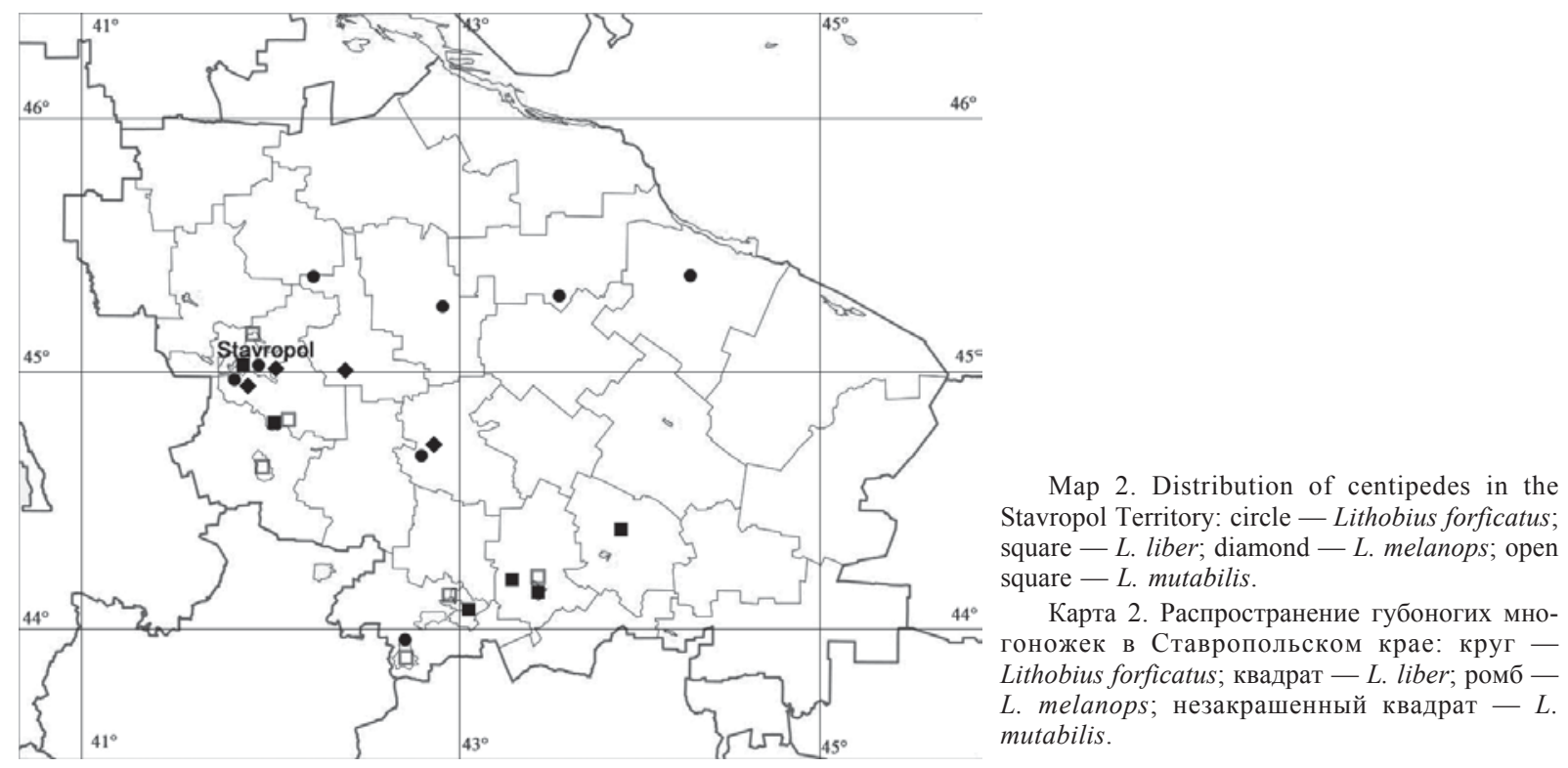

REMARKS. A Caucasian species recorded from Pyatigorsk and Zelenokumsk (Vorontsovo-Aleksandrovskoe) in the Stavropol Territory. In addition, it is also known from the Republic of Adygea, the Krasnodar Territory, the Republic of North Ossetia - Alania and the Republic of Dagestan [Lignau, 1903; Muralewicz, 1926, 1929].

A female gonopod is illustrated to document the identity (Fig. 4).

\section{Lithobius (Lithobius) melanops Newport, 1845} Figs 5-8, Map 2.

MATERIAL. 1 q (ZMUM), Stavropol Territory, Stavropol City, Botanical Garden, 26.III.2014; 1 \& (ZMUS), same locality, 10.IV.2014; $1 \mathrm{O}^{7}$ (ZMUS), same locality, 26.IV.2014; $10^{7}$ (ZMUM), same locality, 25.III.2015; $1 \sigma^{\top}$ (ZMUS), Shpakovsky Distr., near Grushevy, bank of Lake Kravtsovo, 4.X.2014; $1 \sigma^{\top}$ (ZMUM), Grachevsky Distr., near Beshpagir, Pinus plantation, 26.IV.2014; 1 o (ZMUM), Alexandrovsky Distr., $3 \mathrm{~km} \mathrm{~W}$ of Sadovoe, forest belt, 26.IV.2014, all leg. R.V. Zuev.

REMARKS. A European species, in the territory of Russia known from the Republic of Karelia and the Moscow Region [Zalesskaja, 1978]. This is the first formal record of L. melanops in the Caucasus. We have found it only in man-made forest plantations.

New illustrations are provided to document the identity (Figs 5-8).

\section{Lithobius (Lithobius) mutabilis L. Koch, 1862 Map 2.}

MATERIAL. $3 O^{7} \sigma^{7}, 1$, 1 juv. (ZMUS), Stavropol Territory, Stavropol City, Tamansky Forest, 27.VII.2010; $10^{7}, 1$ ㅇ (ZMUM), same locality, 13.X.2012; 1 + (ZMUS), same locality, 7.II.2013; 1 $\sigma^{7}$ (ZMUS), same locality, 10.IX.2013; $1 \sigma^{7}$ (ZMUS), same locality, 11.I.2014; 2 우 (ZMUS), same locality, 15.II.2014; 1 ㅇ (ZMUS), Mamaysky Forest, 27.X.2012; 1 \& (ZMUS), same locality, 3.VIII.2013; 1 + (ZMUM), same locality, 28.IX.2013; $1 \mathrm{O}^{\text {T}}$ (ZMUS), same locality, Pinus sylvestris plantations, 15.II.2014; 1 juv. (ZMUS), Russky Forest, 6.IV.2013; $1 \sigma^{7}$ (ZMUS), same locality,
12.X.2013; $10^{\top}$ (ZMUS), same locality, 7.VI.2014; 2 우 (ZMUS), same locality, 31.I.2015; $3 \sigma^{7} \sigma^{7}, 1$ (ZMUM), Shpakovsky Distr., $5 \mathrm{~km} \mathrm{SSE}$ of Izveshchatelnyi, Strizhament Mountain, forest, 11.VII.2014; 2 O OT $^{7}$ (ZMUS), Kochubeevsky Distr., Nevinnomyssk, confluence of Bolshoy Zelenchuk and Kuban rivers, floodplain forest, 7.IX.2014; $1 \mathrm{O}^{7}$ (ZMUS), Georgievsky Distr., Georgievsk, Safonovsky Forest, 10.VIII.2013, all leg. R.V. Zuev.

REMARKS. A Central European species recorded from Stavropol, Kislovodsk and Zheleznovodsk in the Stavropol Territory [Sseliwanoff, 1881; Muralewicz, 1907, 1929].

Lithobius (Lithobius) peregrinus Latzel, 1880 Figs 9, 10, Map 3.

MATERIAL. 1 (ZMUS), Stavropol Territory, Stavropol City, Tamansky Forest, 15.IX.2012; $1 \mathrm{O}^{7}, 2$ ㅇ (ZMUM), same locality, 14.IV.2013; 1 juv. (ZMUS), same locality, 11.I.2014; $1 \mathrm{O}^{\mathrm{T}}, 2$ 우 (ZMUS), same locality, 18.I.2014; 5 Or $^{7}, 1$ + 4 juv. (ZMUS), Mamaysky Forest, 31.III.2013; $2 \mathrm{O}^{7} \sigma^{7}, 2$ 우 (ZMUS), same locality, 29.VI.2013; $4 \bigcirc^{7} O^{7}, 3$ ㅇ (ZMUM), same locality, 3.VIII.2013; $7 \mathrm{O}^{\top} \mathrm{O}^{\top}, 2$ 우 (ZMUM), same locality, 28.XI.2013; $1 \mathrm{O}^{\top}, 1$ 우, 1 juv. (ZMUM), same locality, 16.III.2014; $10^{7}$ (ZMUS), Tatarsky Forest, 3.V.2013; $2 \bigcirc^{\top} \sigma^{\top}, 4$ 우 (ZMUS), Russky Forest, 6.IV.2013; 1 $\sigma^{7}, 1$ juv. (ZMUS), same locality, 6.VII.2013; 1 O $^{\text {T }}$ (ZMUS), same

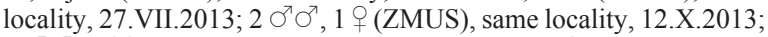
$2 O^{7} \sigma^{7}, 6$ 오 (ZMUS), same locality, 10.V.2014; 19,3 juv. (ZMUS), same locality, 7.VI.2014; $1 \mathrm{O}^{\top}$ (ZMUM), Shpakovsky Distr., 5 km SSE of Izveshchatelnyi, Strizhament Mountain, forest, 14.VII.2013; $1 \sigma^{7}$ (ZMUS), same locality, 9.VII.2014; $4 \sigma^{7} \sigma^{7}, 3$ 오, 9 juv. (ZMUM), Kochubeevsky Distr., Nevinnomyssk, confluence of Bolshoy Zelenchuk and Kuban rivers, floodplain forest, 7.IX.2014; $1 \mathrm{O}^{7}, 1$ \& (ZMUM), Predgornyi Distr., near Pyatigorsk, Beshtau Mountain, forest, 21.VII.2013, all leg. R.V. Zuev.

REMARKS. An eastern Mediterranean species, in the territory of the former Soviet Union known only from the Caucasus [Zalesskaja, 1973, 1978]. In the Stavropol Territory, it is common in forest habitats.

It seems likely that Lithobius (Lithobius) viriatus Sseliwanoff, 1881, recorded from near Kislovodsk and Zheleznovodsk [Sseliwanoff, 1881; Muralewicz, 1929], eventually represents $L$. peregrinus, because $\mathrm{Mu}-$ ralewicz mistakenly considered $L$. peregrinus a syn- 
Figs 5-8. Lithobius melanops Newport, 1845. 5 - habitus, dorsal view; 6 - distal part of last left leg; 7 - coxosternite of forcipular segment; 8 - right female gonopod, mesal view. Pictures taken not to scale.

Рис. 5-8. Lithobius melanops Newport, 1845. 5 - общий вид со спины; 6 - дистальная часть конечной левой ноги; 7 - коксостернит ногочелюсти; 8 - правый гонопод самки, изнутри. Фотографии без масштаба.

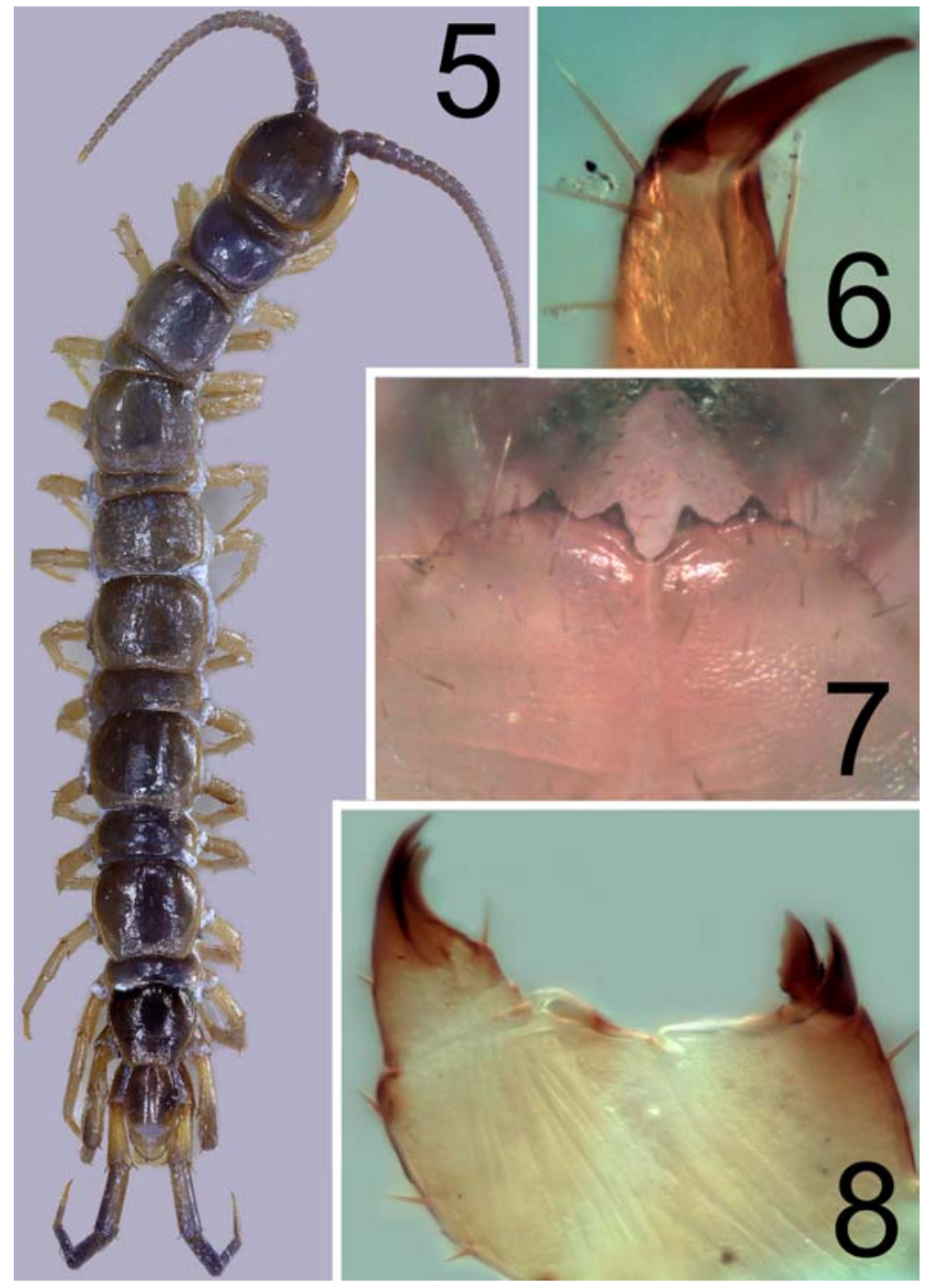

onym of $L$. viriatus. It is impossible to distinguish reliably L. viriatus var. similis Sseliwanoff, 1881 (described from Zheleznovodsk) and L. peregrinus based only on Sseliwanoff's [1881] original description. We have found specimens from near Pyatigorsk $(12 \mathrm{~km}$ south of Zheleznovodsk) certainly to represent $L$. peregrinus.

In addition, Zapparoli [1992] noted that Lignau [1903] had mistakenly reported $L$. viriatus from the Black Sea coast of the Caucasus, the figures in Lignau's paper certainly depicting $L$. peregrinus.

Several illustrations are provided to document the identity (Figs 9, 10).

\section{Lithobius (Lithobius) rufus Muralevitch, 1926} Figs 11-14, Map 3.

MATERIAL. $1 \overbrace{}^{7}$ (ZMUS), Stavropol Territory, Stavropol City, Russky Forest, 31.V.2010, leg. Nikulin S.S.; 1 ○', 2 juv. (ZMUS), same locality, 6.VII.2012; 1 क (ZMUS), same locality, 6.IV.2013; 1 $\sigma^{7}$ (ZMUS), same locality, 27.VII.2013; 1 (ZMUS), same locality, 12.X.2013; 1 ○ (ZMUS), same locality, 3.XI.2013; 2 우, 1 juv. (ZMUS), same locality, 9.III.2014; $2 \sigma^{\top} \sigma^{\top}, 2$ 우 (ZMUM), same

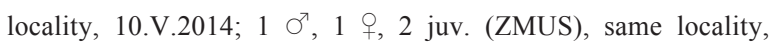
7.VI.2013; 2 O $^{7}$ (ZMUS), Tamansky Forest, 13.X.2012; $1 \sigma^{7}$ (ZMUS), same locality, 5.II.2013; $1 \sigma^{7}$ (ZMUS), same locality, 7.II.2013; 1 , 2 juv. (ZMUS), same locality, 21.III.2013; $2 \sigma^{7} \sigma^{7}, 2$ Oᄋ, 2 juv. (ZMUM), same locality, 14.IV.2013; $10^{\top}, 1$ ( same locality, 28.IV.2013; 1 + (ZMUS), same locality, 15.VI.2013; 2 오 (ZMUS), same locality, 10.IX.2013; $1 \sigma^{7}$ (ZMUS), same locality, 12.IX.2013; $1 \sigma^{7}, 1$ ( (ZMUS), same locality, 5.X.2013; 1 $\sigma^{7}$ (ZMUS), same locality, 9.X.2013; 1 juv. (ZMUS), same locality, 7.IV.2014; 1 ㅇ (ZMUS), same locality, 8.IV.2014; $2 \mathrm{O}^{7} \mathrm{O}^{7}, 1$ juv. (ZMUS), same locality, 19.IV.2014; $1 \sigma^{7}, 6$ juv. (ZMUS), same locality, 1.VI.2014; $1 \sigma^{7}$ (ZMUS), Mamaysky Forest, 27.X.2012; 1 ( (ZMUS), same locality, 31.III.2013; $2 \mathrm{O}^{7} \sigma^{7}, 2$ 우 (ZMUS), same locality, 16.IV.2013; $1 \sigma^{7}$ (ZMUS), same locality, 28.IX.2013; 1 juv. (ZMUS), Tatarsky Forest, 3.V.2013; 1 O (ZMUS), same locality, 12.X.2013; $1 \mathrm{O}^{T}$ (ZMUM), Shpakovsky Distr., $5 \mathrm{~km}$ SSE of Izveshchatelnyi, Strizhament Mountain, forest, 3.VI.2010; 1 ( (ZMUS), same locality, 14.VII.2013; $1 \mathrm{sad}+2$, juv. (ZMUM), Georgievsky Distr., Georgievsk, Safonovsky Forest, 10.VIII.2013; $1 \sigma^{7}, 1$ o (ZMUS), Predgornyi Distr., Pyatigorsk, Mashuk Mountain, forest, 20.VII.2013; 1 (ZMUS), near Kislovodsk, Borgustansky Ridge, steppe, 20.VII.2013; $10^{2}, 1$ ( $($ ZMUM), Kochubeevsky Distr., Nevinnomyssk, confluence of Bolshoy Zelenchuk and Kuban rivers, floodplain forest, 7.IX.2014, all leg. R.V. Zuev.

REMARKS. A Caucasian species described from 


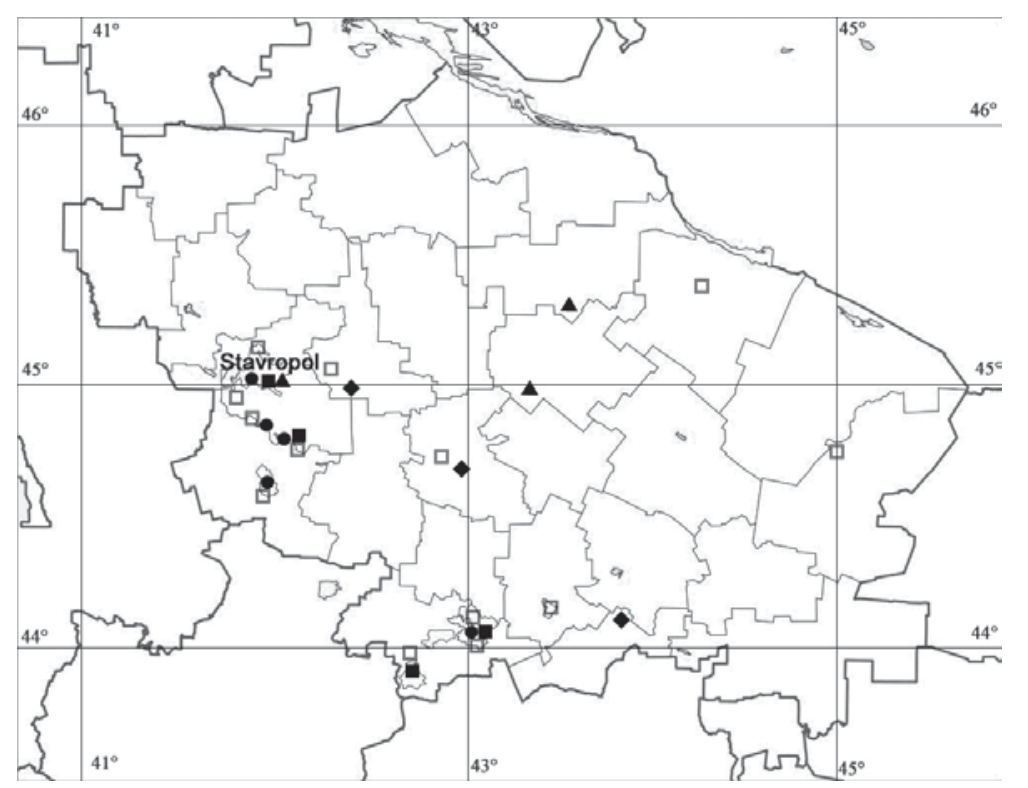

Map 3. Distribution of centipedes in the Stavropol Territory: circle - Lithobius peregrinus; square - L. rufus; diamond - L. crassipes; open square - L. ferganensis; triangle Geophilus flavus.

Карта 3. Распространение губоногих многоножек в Ставропольском крае: круг Lithobius peregrinus; квадрат - L. rufus; ромб - L. crassipes; незакрашенный квадрат — L. ferganensis; треугольник — Geophilus flavus.
Makhachkala (Petrowsk), Republic of Dagestan, also recorded from Stavropol [Muralewicz, 1926, 1929].

A usual forest-dwelling species in the region.

The samples we have found show rugose tergites, while Muralewicz stated they were smooth. However, the above new specimens fit the original description in all other details. Another Caucasian species which also has rugose tergites, Lithobius (Lithobius) foviceps $\mathrm{Mu}-$ ralewicz, 1926, is known from Azerbaijan (Kemervan), but it is larger and shows more numerous ocelli and coxal pores.

New illustrations are provided to document the identity (Figs 11-14).

Lithobius (Lithobius) stuxbergii Sseliwanoff, 1881

REMARKS. This species was originally described from near Kislovodsk; in addition, it was listed from the Krasnodar Territory, the Republic of North Ossetia - Alania, the Karachayevo-Cherkessia Republic, Abkhazia and the Crimea [Sseliwanoff, 1881; Lignau, 1903; Muralewicz, 1907, 1927, 1929; Zalesskaja, 1973, 1978].
We have failed to find L. stuxbergii in the Stavropol Territory.

Lithobius (Monotarsobius) crassipes L. Koch, 1862 Map 3.

MATERIAL. $1 \sigma^{7}, 41$ juv. (ZMUS), Stavropol Territory, Kirovsky Distr., near Kommayak, forest belt, soil, 10.VII.2013; 1 ㅇ (ZMUS), Grachevsky Distr., near Beshpagir, Pinus plantation, 26.IV.2014; 2 90 (ZMUM), $1 \mathrm{~km} \mathrm{SW}$ of Beshpagir, forest, 17.VII.2014; $1 \mathrm{O}^{7}$ (ZMUS), Alexandrovsky Distr., near Alexandrovskoe, steppe, 26.IV.2014, all leg. R.V. Zuev.

REMARKS. A western Palaearctic species, in the Caucasus recorded from Azerbaijan, Georgia, the Republic of Adygea and the Krasnodar Territory [Lignau, 1903; Zalesskaja, 1973]. It is rare in the region, mainly occurring in man-made forest plantations.

Lithobius (Monotarsobius) curtipes C.L. Koch, 1847 Map 1.

MATERIAL. $40^{7} \sigma^{7}, 4$ + 9,2 juv. (ZMUS), Stavropol Territory, Stavropol City, Tamansky Forest, soil, 27.VII.2010; 4 ○ $^{\top} \sigma^{7}, 1$ ㅇ
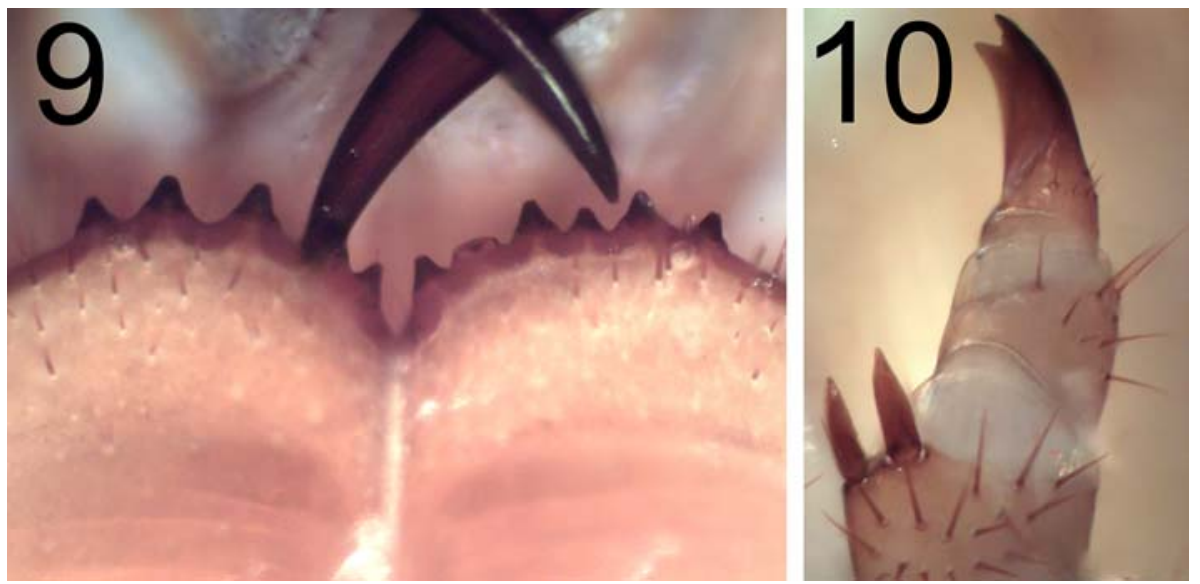

Figs 9-10. Coxosternite of forcipular segment and right female gonopod of Lithobius peregrinus Latzel, 1880, ventral and lateral views, respectively. Pictures taken not to scale.

Рис. 9-10. Коксостернит ногочелюсти и правый гонопод самки у Lithobius peregrinus Latzel, 1880, соответственно снизу и сбоку. Фотографии без масштаба. 


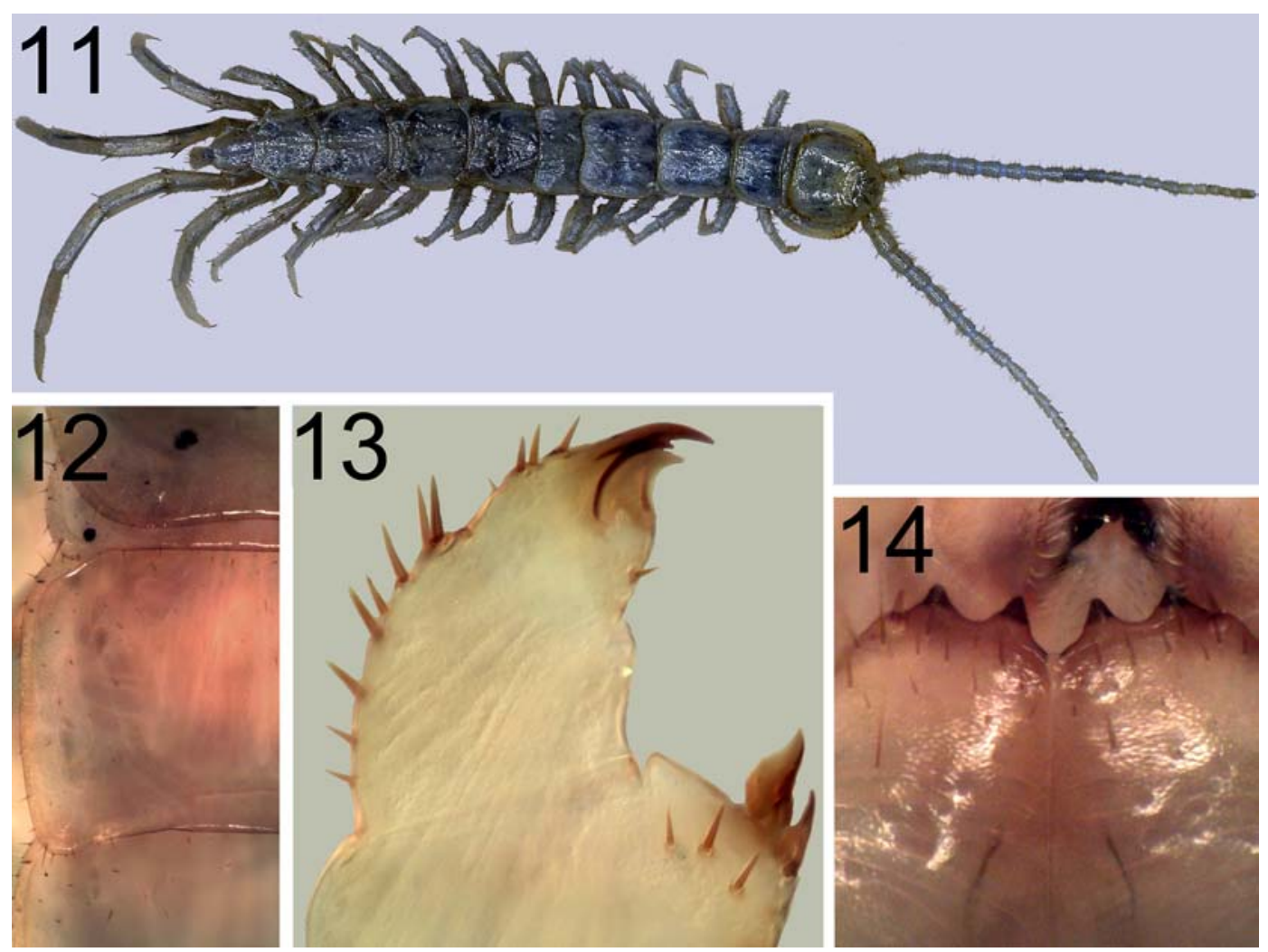

Figs 11-14. Lithobius rufus Muralewicz, 1926. 11 - habitus, dorsal view; 12 - tergites VI and VII; 13 - right female gonopod, mesal view; 14 - coxosternite of forcipular segment. Pictures taken not to scale.

Рис. 11-14. Lithobius rufus Muralewicz, 1926. 11 - общий вид со спины; 12 - тергиты VI и VII; 13 - правый гонопод самки, изнутри; 14 - коксостернит ногочелюсти. Фотографии без масштаба.

(ZMUS), same locality, soil, 8.IX.2012; 2 우 (ZMUS), same locality, 15.IX.2012; $1 \mathrm{O}^{7}$ (ZMUS), same locality, 5.II.2013; 2 O $^{7} \mathrm{O}^{7}, 1$ +, 2 juv. (ZMUS), same locality, 21.III.2013; 1 + (ZMUS), same locality, 14.IV.2013; 1 우 (ZMUS), same locality, 28.IV.2013; 2 우오

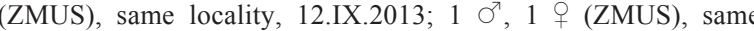
locality, 5.X.2013; $1 \bigcirc^{\top}, 1$ \& (ZMUS), same locality, 9.X.2013; 1 $\sigma^{2}$ (ZMUS), same locality, 18.I.2014; $1 \mathrm{O}^{7}, 4$ 우 (ZMUS), same locality, 15.II.2014; 1 O' (ZMUS), Mamaysky Forest, 6.II.2013; 3 우 (ZMUS), same locality, 31.III.2013; $2 \mathrm{O}^{7} \mathrm{O}^{7}, 1$ juv. (ZMUS), same locality, 16.IV.2013; 2 Oᄋ, 1 juv. (ZMUS), same locality, 29.VI.2013; 1 O $^{7}, 3$ 우, 1 juv. (ZMUS), same locality, 3.VIII.2013; $1 \sigma^{7}, 1$ ( (ZMUS), same locality, 28.IX.2013; $5 \sigma^{7} \sigma^{7}, 3$ 우 (ZMUS), same locality, soil, 16.III.2014; $3 \mathrm{O}^{2} \mathrm{O}^{\mathrm{x}}$, 6 우 (ZMUM), Russky Forest, 23.III.2013; 3 O $^{7}, 6$ +P (ZMUS), same locality, soil, 6.IV.2013; $1 \mathrm{O}^{\top}, 2$ 우, 1 juv. (ZMUS), same locality, 6.VII.2013; 2 오, 1 juv. (ZMUS), same locality, 27.VII.2013; $1 \sigma^{7}$ (ZMUS), same locality, 12.X.2013; 1 (ZMUS), same locality, 3.XI.2013; 1 ㅇ (ZMUS), same locality, 2.III.2014; $60^{7} 0^{7}, 5$ 오 (ZMUM), same locality, 9.III.2014; $2 \mathrm{O}^{7} \mathrm{O}^{7}, 3$ क्+, 3 juv. (ZMUS), same locality, 5.V.2014; $1 \sigma^{7}, 1$ juv. (ZMUS), same locality, 10.V.2014; $2 \sigma^{7} \sigma^{7}$, 2 Oᄋ 1 juv. (ZMUS), same locality, 7.VI.2014; 1 ㅇ (ZMUS), Tatarsky Forest, 3.V.2013; 1 (ZMUS), same locality, 23.IX.2013; 2 O $^{\top} \sigma^{\top}$ (ZMUS), same locality, 19.X.2013; 1 O $^{\top}$ (ZMUS), Botanical Garden, Pinus plantation, 6.III.2014; $2 O^{7} \sigma^{7}, 1$ 1,1 juv. (ZMUM), same locality, Picea plantation, 7.VI.2014; 2 +क, 1 juv. (ZMUS), Shpakovsky Distr., near Grushevy, steppe, 23.V.2013; $3 O^{7} O^{7}$, 3 우, 1 juv. (ZMUS), same locality, 26.X.2013; $3 \mathrm{O}^{7} \sigma^{7}, 3$ 우 (ZMUS), same locality, 23.III.2014; 2 O' $\mathcal{O}^{7}, 1$ ㅇ (ZMUS), same locality, 14.VI.2014; $10^{7}, 2$ 오 (ZMUS), same locality, 4.X.2014; $10^{\circ}$ (ZMUS), near Tatarka, steppe, 27.V.2013; $1 \bigcirc^{7}, 2$ ㅇ, 2 juv. (ZMUS), $5 \mathrm{~km}$ SSE of Izveshchatelnyi, Strizhament Mountain, forest, 14.VII.2013; 1 O $^{7}$ (ZMUS), same locality, steppe, 14.VII.2013; $2 \mathrm{O}^{\top} \mathrm{O}^{\top}, 2$, + (ZMUS), same locality, forest, 9.VII.2014;
1 , 3 juv. (ZMUS), near Rynok, Nedremannaya Mountain, forest, soil, 19.IV.2013; $10^{7}, 1$, 1 juv. (ZMUS), same locality, 15.VII.2014; 1 + (ZMUS), Grachevsky Distr., 1 km SW of Beshpagir, forest, 17.VII.2014; 2 OO, 3 juv. (ZMUS), Alexandrovsky Distr., near Severnoe, forest belt, 26.IV.2014; $2 \mathrm{O}^{\top} \sigma^{\top}, 3$ 우, 3 juv.

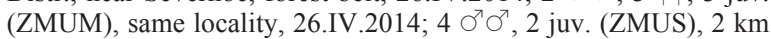
NE of Kruglolesskoe, forest belt, 26.IV.2014; 1 o (ZMUS), near Kruglolesskoe, pasture, 26.IV.2014; $1 \sigma^{7}, 1$, 4 juv. (ZMUS), Andropovsky Distr., $3 \mathrm{~km} \mathrm{~S}$ of Novy Yankul, forest belt, 12.IV.2014; 1 ( (ZMUS), Kochubeevsky Distr., Nevinnomyssk, confluence of Bolshoy Zelenchuk and Kuban rivers, floodplain forest, 7.IX.2014; 1 juv. (ZMUS), Petrovsky Distr., near Lake Solyonoe, steppe, 10.VII.2014; 2 우 (ZMUS), Arzgirsky Distr., near Arzgir, steppe, in manure, 2.V.2014; 2 OO (ZMUS), Georgievsky Distr., near Alexandriyskaya, forest, 11.VIII.2013; $10^{7}, 1$ (ZMUS), Predgornyi Distr., Pyatigorsk, Mashuk Mountain, forest, 20.VII.2013; 1 , 3 juv. (ZMUS), near Pyatigorsk, Beshtau Mountain, forest, 21.VII.2013; $1 O^{7}, 4$ juv. (ZMUS), near Kislovodsk, Borgustansky Ridge, forest, 20.VII.2013; $1 \mathrm{O}^{7}, 1$, 1 juv. (ZMUS), Budennovsky Distr., near Terek, Kuma river, floodplain forest, 10.V.2015, all leg. R.V. Zuev; $5 \sigma^{7} \sigma^{7}, 1$ juv. (ZMUS), Kislovodsk, nest of Turdus merula, VI.2000; 4 juv. (ZMUS), same locality, nest of Micromys minutus, 3.VII.2011, all leg. V.A. Telpov; $10^{7}$ (ZMUS), Izobilnensky Distr., near Rogdestvenskaya, 18.V.2006; 1 O (ZMUS), near Moskovskoe, forest, 19.IV.2008; $2 \sigma^{7} \sigma^{7}, 2$ 우, 1 juv. (ZMUS), same locality, 11.VII.2008; 1 9 (ZMUS), Novoalexandrovsky Distr., near Temigbeksky, forest belt, 19.IV.2008, all leg. M.B. Patyuta.

REMARKS. A Central Asian - European species widely distributed across Russia: the entire European part, including the Arctic Circle, to the Caucasus in the south, up to the Altai Mts in the east [Zalesskaja, 1973, 


\section{5}

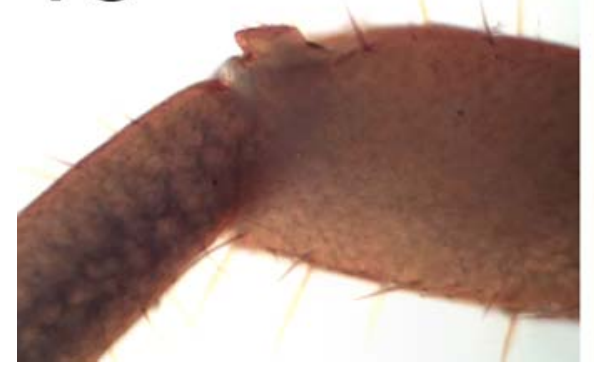

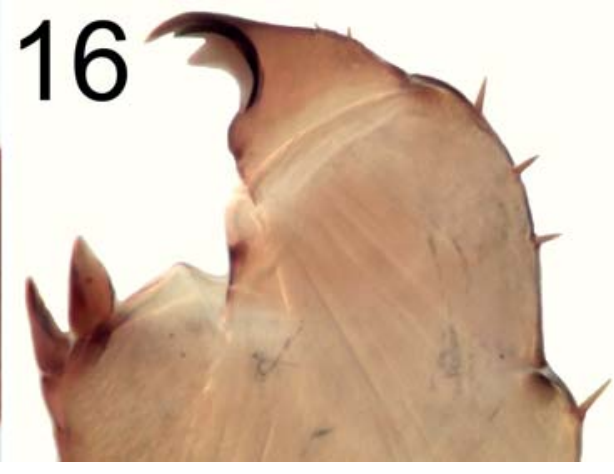

Figs 15-16. Distal part of right tibia $15\left(\mathrm{O}^{7}\right)$ and left gonopod (+) of Lithobius ferganensis (Trotzina, 1894), lateral and mesal views, respectively. Pictures taken not to scale.

Рис. 15-16. Дистальная часть голени правой 15 ноги $\left(\mathrm{O}^{7}\right)$ и левый гонопод $(+)$, у Lithobius ferganensis (Trotzina, 1894), соответственно сбоку и изнутри. Фотографии без масштаба.
1978]. Muralewicz [1907, 1926, 1927, 1929] reported L. curtipes from Pyatigorsk and Kislovodsk.

There had been confusion caused by different understandings of the phrase "einer kurzen kegelförmigen Fortsatz" concerning the tibial projection on the $15^{\text {th }}$ pair of legs in the description of $L$. curtipes as given by Koch [1862] until Eason [1972] provided a redescription of the neotype of L. curtipes. Thus, Muralewicz's records, in the opinion of Zalesskaja, may have actually referred to L. ferganensis [Zalesskaja, 1978].

According to our data, L. curtipes inhabits the whole region, occurring in all habitats except semi-desert ones. Interestingly, it has been found in the nests of Turdus merula and Micromys minutus. This is the most common and abundant representative of Lithobiomorpha in the study area. 1894)

Lithobius (Monotarsobius) ferganensis (Trotzina,

Figs 15, 16, Map 3.

MATERIAL. $1 \sigma^{T}$ (ZMUS), Stavropol Territory, Stavropol City, Botanical Garden, 10.X.2012; $3 \mathrm{O}^{\top} \mathrm{O}^{\top}, 2$ 우 (ZMUS), same locality, 6.III.2014; 6 O $^{7} \sigma^{7}, 4$ 우 (ZMUS), same locality, 26.III.2014; $5 \mathrm{O}^{7} \mathrm{O}^{7}, 3$ 우, 2 juv. (ZMUM), same locality, 10.IV.2014; 1 古 (ZMUS), Tamansky Forest, 15.IX.2012; $1 \bigcirc^{7}, 5$ 우 (ZMUM), same locality, 13.X.2012; 1 ㅇ (ZMUS), same locality, 13.X.2012; 4 우 (ZMUS), same locality, 21.III.2013; $1 \sigma^{7}, 1$ क (ZMUS), same locality, 14.IV.2013; $1 \sigma^{7}, 4$ ㅇ (ZMUS), same locality, 28.IV.2013; $1 \mathrm{O}^{\top}, 1$ + (ZMUS), same locality, 5.X.2013; $1 \mathrm{O}^{7}$ (ZMUS), same locality, 11.I.2014; $4 \sigma^{7} \sigma^{7}$ (ZMUS), same locality, 18.I.2014; 2 $\sigma^{\top} \sigma^{7}, 3$ 우, 1 juv. (ZMUS), same locality, 26.III.2014; $30^{7} \sigma^{\top}, 1$, 1 , 4 juv. (ZMUS), Mamaysky Forest, 31.III.2013; 1 (ZMUS), same locality, 29.VI.2013; $2 \sigma^{7} \sigma^{7}$ (ZMUS), same locality, 28.IX.2013; 2 +P (ZMUS), same locality, Pinus plantations, 27.X.2012; 3 O $^{7} \mathrm{O}^{7}, 8$ 우 (ZMUS), Russky Forest, 23.III.2013; $1 \sigma^{7}, 3$ juv. (ZMUS), same locality, 6.VIII.2013; 1 ( (ZMUS), same locality, 27.VII.2013; $1 \mathrm{O}^{7}, 1$ (ZMUS), same locality, 12.X.2013; $2 \mathrm{O}^{7} \sigma^{7}, 3$ 우, 1 juv (ZMUM), same locality, 3.XI.2013; 3 우 (ZMUS), same locality,

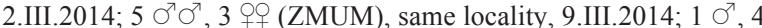
우 (ZMUS), same locality, 4.V.2014; $10^{7}, 5$ 우, 5 juv. (ZMUS), same locality, 10.V.2014; $3 \bigcirc^{7} \sigma^{7}, 4$ 오 (ZMUS), same locality, 6.VI.2014; $3 \bigcirc^{7} \sigma^{7}$ (ZMUS), same locality, 31.I.2015; $1 \bigcirc^{\top}$ (ZMUS), Tatarsky Forest, 19.X.2013; $2 O^{7} \sigma^{7}$ (ZMUS), Shpakovsky Distr., near Grushevyi, steppe, 23.V.2013; 1 + (ZMUS), same locality, 26.X.2013; 7 O $^{7}, 11$ 우, 2 juv. (ZMUM), same locality, 23.III. 2014; $10^{7}, 2$ 우 (ZMUM), same locality, 14.VI.2014; $1 \mathrm{O}^{7}, 4$ 우우 (ZMUS), same locality, 4.X.2014; $1 \sigma^{7}, 1$ + (ZMUS), near Rynok, Nedremannaya Mountain, forest, 19.IV.2013; $2 \bigcirc^{7} O^{7}, 1$, 2 juv (ZMUS), Kochubeevsky Distr., Nevinnomyssk, confluence of Bolshoy Zelenchuk and Kuban rivers, floodplain forest, 7.IX.2014; 1 (ZMUS), Grachevsky Distr., $5 \mathrm{~km}$ ENE of Staromarevka,
26.IV.2014; 1 q (ZMUS), Alexandrovsky Distr., $7 \mathrm{~km} \mathrm{WNW} \mathrm{of}$ Alexandrovskoe, forest belt, 26.IV.2014; $1 O^{7}$ (ZMUM), Arzgirsky Distr., near Arzgir, steppe, 1.V.2014; $10^{7}$ (ZMUS), Georgievsky Distr., Georgievsk, Safonovsky Forest, 10.VIII.2013; $1 \sigma^{7}, 1$ O (ZMUS), Predgornyi Distr., Pyatigorsk, Mashuk Mountain, forest, 20.VII.2013; 1 + 1 juv. (ZMUS), near Pyatigorsk, Beshtau Mountain, forest, 21.VII.2013; 1 ( (ZMUS), near Kislovodsk, Borgustansky Ridge, steppe, 20.VII.2013; 1 O', 1 + (ZMUS), Neftekumsky Distr., near Neftekumsk, Kamysh-Burunsky foreSt, floodplain of Kuma River, 24.V.2015, all leg. R.V. Zuev; 1 (ZMUS), Kislovodsk, nest of Turdus merula, VI.2000, leg. V.A. Telpov.

REMARKS. A Turano-European species. According to Zalesskaja [1978], the reports by Sseliwanoff and Muralewicz of L. curtipes and L. pusillus Sseliwanoff, 1881 [Sseliwanoff, 1881; Muralewicz, 1907, 1926, 1929] in the Caucasus (including Pyatigorsk) must have actually concerned $L$. sseliwanoffi Garbowski, 1897. Eason [1997], in his turn, synonymized $L$. sseliwanoffi with the extremely variable species $L$. ferganensis, and we agree with his opinion.

The distribution L. ferganensis in the region is the same as that of L. curtipes, also found in the nests of Turdus merula.

New illustrations are provided to document the identity, including the characteristic dorsodistal wart on tibia 15 (Figs 15, 16).

\section{ORDER SCOLOPENDROMORPHA Family SCOLOPENDRIDAE}

\section{Scolopendra cingulata Latreille, 1789} Figs 17-20, Map 4.

MATERIAL. 1 subad., 1 juv. (ZMUS), Stavropol Territory, Shpakovsky Distr., near Grushevy, steppe, 23.V.2013; 3 juv. (ZMUM), same locality, 14.VI.2014; 1 О (ZMUS), Grachevsky Distr., near Tuguluk, steppe, 21.V.2013; 1 \& (ZMUM), Alexandrovsky Distr., near Alexandrovskoe, steppe, 26.IV.2014; $1 \sigma^{7}$ (ZMUM), Arzgirsky Distr., near Arzgir, steppe, 1.V.2014; 1 ○ (ZMUS), same locality, 1.V.2014; 1 sad. (ZMUS), Petrovsky Distr., near Lake Solyonoe, steppe, 10.VII.2014; 1 sad. (ZMUS), Levokumsky Distr., near Urozhaynoe, floodplain of Kuma river, in soil, 24.V.2015, all leg. R.V. Zuev; 1 (ZMUS), Sovetsky Distr., near Zelenokumsk, indoors, 21.VI.2009, leg. B.K. Kotti; 1 య (ZMUS), Apanasenkovsky Distr., near Belye Kopani, steppe, 28.V.2004, leg., S.A. Masianova \& L.B. Rushchina; 1 juv. (ZMUS), same locality, 1.VI.2004, leg. A. Parashchuk \& L. Startseva; 1 ㅇ (ZMUS), Turkmensky Distr., Letnyaya Stavka, 28.V. 2014, leg. K.S. Priymak.

REMARKS. A Mediterranean species widespread in the Caucasus, known from Georgia, the Republic of 


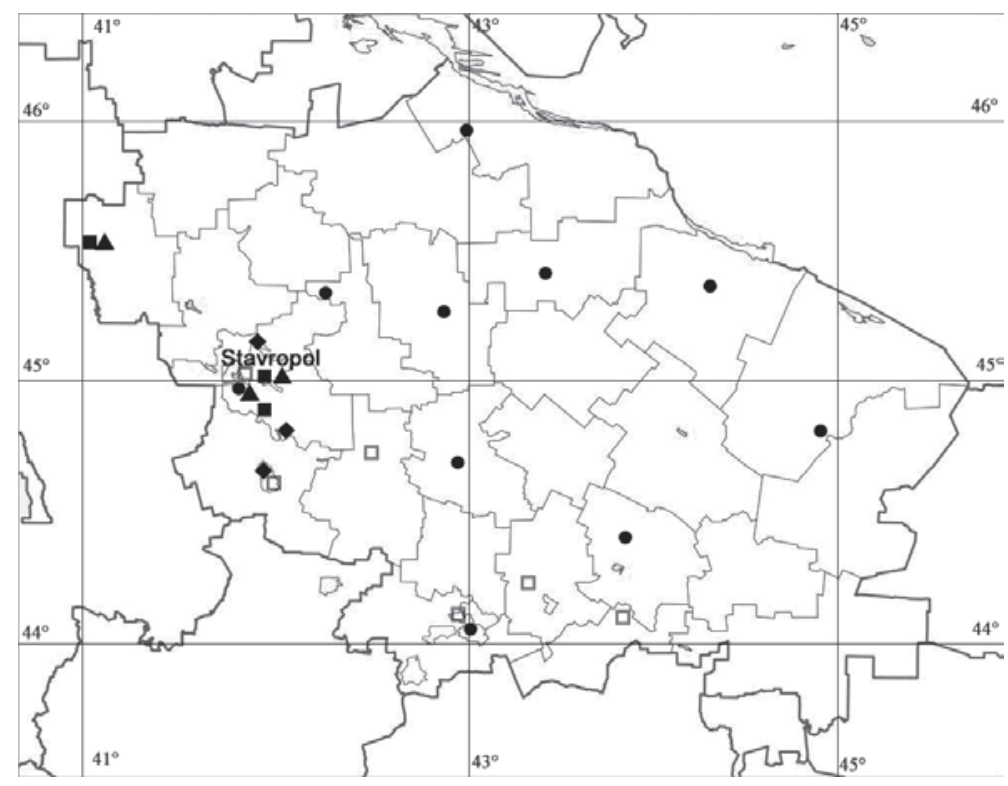

Map 4. Distribution of centipedes in the Stavropol Territory: circle - Scolopendra cingulata; square - Cryptops caucasius; diamond - C. hortensis; open square - Henia bicarinata; triangle - Strigamia aff. caucasia.

Карта 4. Распространение губоногих многоножек в Ставропольском крае: круг Scolopendra cingulata; квадрат - Cryptops caucasius; ромб - C. hortensis; незакрашенный квадрат - Henia bicarinata; треугольник - Strigamia aff. caucasia.
Dagestan, the Chechen Republic and the Krasnodar Territory [Brandt, 1840; Kessler, 1875; Lignau, 1903; Zalesskaja, Schileyko, 1991, 1992]. In the Stavropol Territory, it has been recorded from Pyatigorsk [Sseliwanoff, 1883; Muralewicz, 1907] and Stavropol (Voroshilovsk) [Reznik, 1940]. In Reznik's paper, it is referred to as Scolopendra sp. This species occurs in steppe habitats across the region.

New illustrations are provided to document the identity (Figs 17-20).

\section{Family CRYPTOPIDAE}

\section{Cryptops (Cryptops) caucasius Verhoeff, 1934} Figs 21, 22, Map 4.

MATERIAL. 3 ad. (ZMUS), Stavropol Territory, Stavropol City, in wet sand, 23.V.2014; 1 ad. (ZMUM), same locality, 19.IV 2015, all leg. S.I. Sigida; 1 ad. (ZMUS), Mamaysky Forest, 13.VII 2011; 1 ad., 2 juv. (ZMUM), same locality, 27.X.2012; 1 juv. (ZMUS), same locality, 16.III.2014; 1 juv. (ZMUS), same locality, 27.IX.2014; 2 ad. (ZMUS), Tamansky Forest, 11.I.2014; 1 ad. (ZMUS), same locality, 18.I.2014; 3 ad. (ZMUM), same locality, 15.II.2014; 1 ad. (ZMUS), same locality, 17.V.2014; 1 ad. (ZMUM), Shpakovsky Distr., Verhneegorlyksky, Lopatin Forest, 9.VI.2012, all leg. R.V. Zuev; 1 juv. (ZMUS), Novoalexandrovsky Distr., near Temigbeksky, field, soil, 19.IV.2008, leg. M.B. Patyuta.

REMARKS. A Caucasian species also known from the Crimea and Turkestan [Zalesskaja, Schileyko, 1991, 1992]. Various authors reported $C$. anomalans Newport, 1844 from the Caucasus (including Kislovodsk and Pyatigorsk) [Sseliwanoff, 1883; Lignau, 1903; Muralewicz, 1907, 1926]. In addition, Folkmanová [1958] described the subspecies C. anomalans hirsutus Folkmanova, 1958 from the Krasnodar Territory; so far this subspecies is only known from the holotype. It is noteworthy that Zalesskaja \& Schileyko [1991, 1992] did not report $C$. anomalans from the Caucasus.
So it seems that in the Caucasus $C$. anomalans is replaced by the similar C. caucasius.

We have found specimens identifiable as C. caucasius: the cephalic plate with sutures extending only a short way back from the bases of the antennae and the posterior sutures occupying the posterior half of the cephalic plate; a tridentate labrum (Fig. 21); tergite I with transverse sutures, cruciform sutures being absent (larger specimens can show incomplete paramedian sutures, Fig. 22).

\section{Cryptops (Cryptops) hortensis (Donovan, 1810) Map 4.}

MATERIAL. 2 ad., 1 juv. (ZMUS), Stavropol Territory, Stavropol City, Russky Forest, 14.V.2014; 4 ad., 4 juv. (ZMUM), same locality, 7.VI.2014; 1 ad. (ZMUS), Shpakovsky Distr., 5 km SSE of Izveshchatelnyi, Strizhament Mountain, forest, 14.VII.2013; 1 ad. (ZMUM), Kochubeevsky Distr., Nevinnomyssk, confluence of Bolshoy Zelenchuk and Kuban rivers, floodplain forest, 7.IX.2014, all leg. R.V. Zuev.

REMARKS. A Central Asian - European species. Sseliwanoff [1883] recorded C. bidenticulatus Sseliwanoff, 1883 from Pyatigorsk, a species later recognized as a synonym of $C$. hortensis [Lignau, 1915].

Very likely, the record of $C$. inermipes Pocock, 1888 by Muralewicz [1926] near Pyatigorsk, a species known only from Christmas Island in the Indian Ocean, eventually also represents $C$. hortensis. Both species have approximately the same size and they show no sutures on the first tergite.

C. inermipes is unique in the genus in that, according to Pocock [1888], the ultimate legs lack saw teeth. However, the ultimate legs of in the holotype are missing and Lewis [2011] suggested that Pocock may have mistaken the $20^{\text {th }}$ pair of legs with the ultimate pair [Waldock, Lewis, 2014]. Muralewicz probably made the same mistake. 

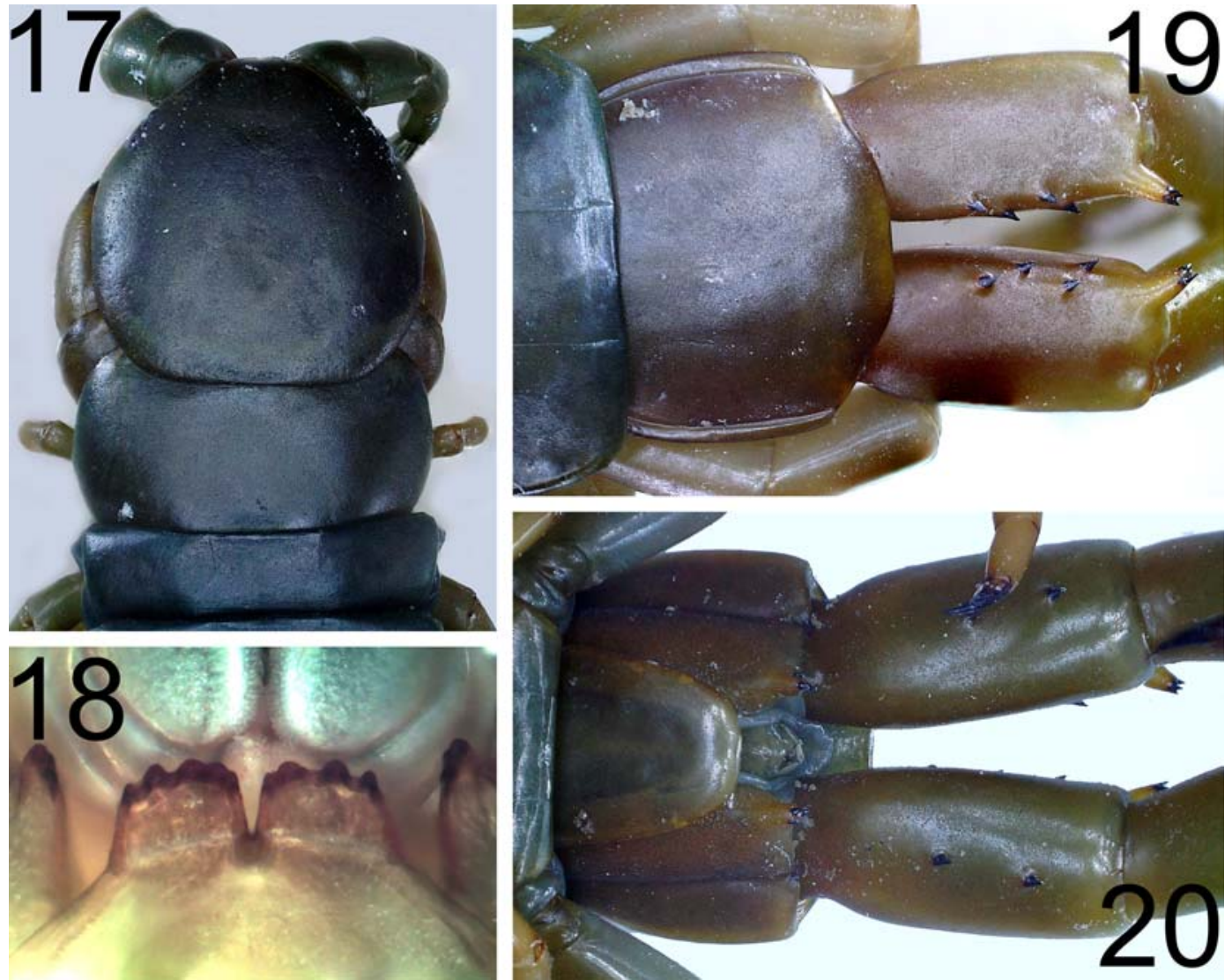

Figs 17-20. Scolopendra cingulata Latreille, 1789. 17 - head and tergite I; 18 - tooth-plate of forcipular coxosternite; $19-$ terminal part of body and prefemur of ultimate leg, dorsal view; 20 - terminal part of body and prefemur of ultimate leg, ventral view. Pictures taken not to scale.

Рис. 17-20 . Scolopendra cingulata Latreille, 1789. 17 — голова и тергит I; 18 - зубная пластинка ногочелюсти; 19 - задний конец тела и префемур конечных ног, вид сверху; 20 - задний конец тела и префемур конечных ног, вид снизу Фотографии без масштаба.

\section{ORDER GEOPHILOMORPHA \\ Family DIGNATHODONTIDAE}

\section{Henia (Henia) illyrica (Meinert, 1870)}

MATERIAL. $1+$ (ZMUS), Stavropol Territory, Stavropol City, Tatarsky Forest, 3.V.2013; 1 q, 1 juv. (ZMUS), Russky Forest, 10.V.2014; 1 O’ $^{7} 4$ 우 (ZMUM), same locality, 26.IV.2015, all leg. R.V. Zuev.

REMARKS. A southern European species, on the Caucasus it has been recorded from the Krasnodar Territory (Novorossiysk) [Lignau, 1903].

\section{Henia (Meinertia) bicarinata (Meinert, 1870) Map 4.}

MATERIAL. 1 (ZMUS), Stavropol Territory, Stavropol City, Mamaysky Forest, Pinus plantation, 27.X.2012; $1 \sigma^{7}$ (ZMUM), Kochubeevsky Distr., Nevinnomyssk, confluence of Bolshoy Zelenchuk and Kuban rivers, floodplain forest, 7.IX.2014; 1 o (ZMUS), Andropovsky Distr., $3 \mathrm{~km} \mathrm{~S}$ of Novy Yankul, forest belt, 12.IV.2014; 1 O (ZMUM), Georgievsky Distr., near Alexandriyskaya, forest, 11.VIII.2013; 1 juv. (ZMUS), Predgornyi Distr., near Pyatigorsk, Beshtau Mountain, forest, 21.VII.2013; 1 o (ZMUM), Kirovsky Distr., near Kommayak, forest belt, soil, 10.VII. 2013, all leg. R.V. Zuev.
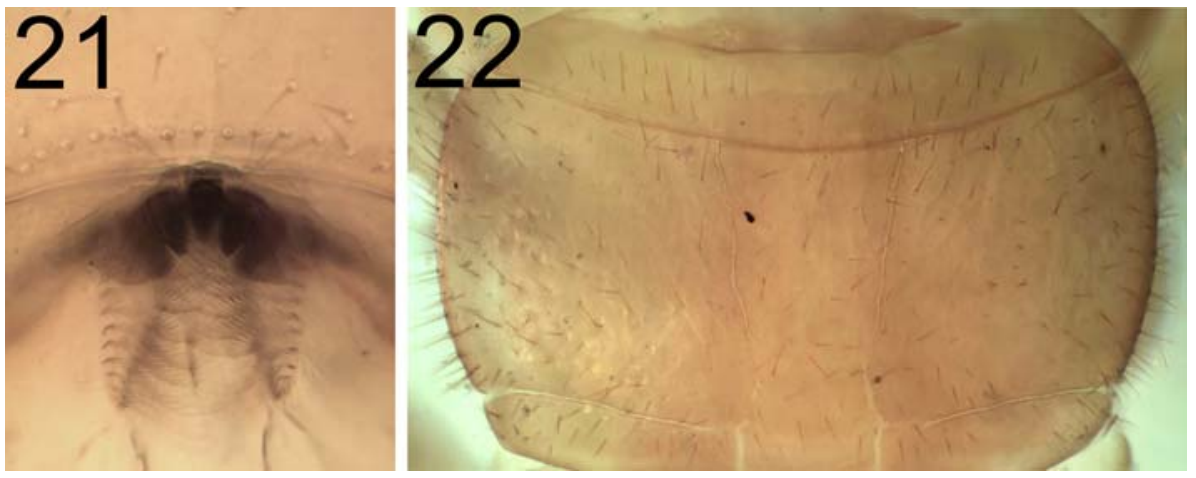

Figs 21-22. Labrum and tergite I of Cryptops caucasius Verhoeff, 1934, respectively. Pictures taken not to scale.

Рис. 21-22. Соответственно лабрум и тергит I у Cryptops caucasius Verhoeff, 1934. Фотографии без масштаба. 

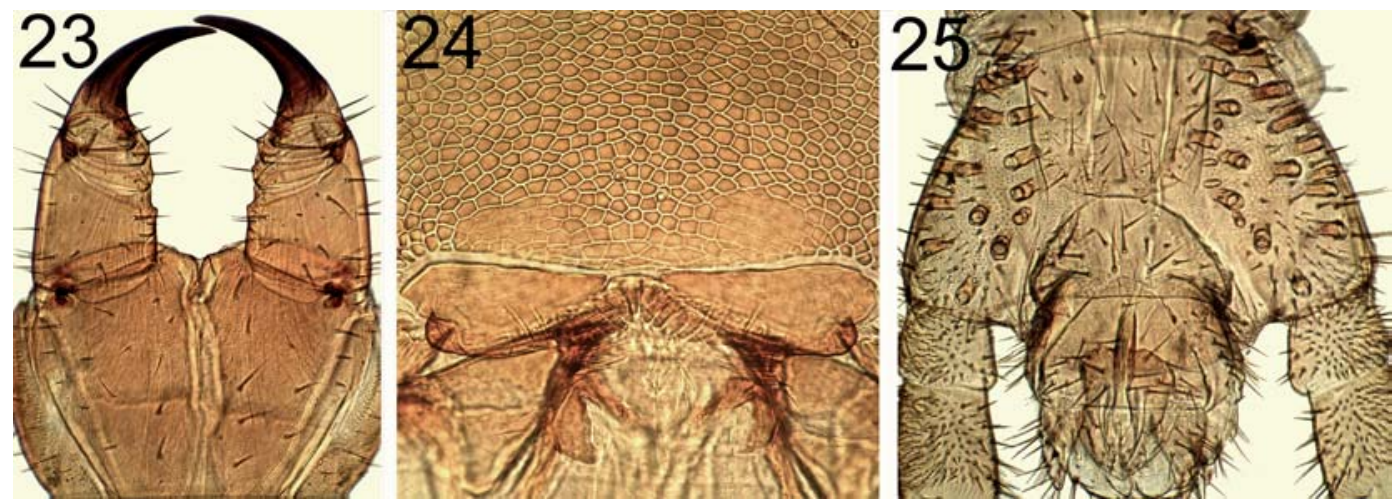

Figs 23-25. Escaryus retusidens Attems, $1904\left(0^{7}\right) .23$ - forcipular segment, ventral view; 24 - labrum; 25 - terminal part of body, ventral view. Pictures taken not to scale.

Рис. 23-25. Escaryus retusidens Attems, 1904 ( (7): 23 - ногочелюсть, снизу; 24 - лабрум; 25 - задний конец тела, снизу. Фотографии без масштаба.

REMARKS. A Mediterranean species, in the Caucasus it has been recorded from Pyatigorsk and Lenkoran, Azerbaijan [Sseliwanoff, 1883; Attems, 1907; Muralewicz, 1907].

\section{Family SCHENDYLIDAE}

Escaryus retusidens Attems, 1904

Figs 23-25.

MATERIAL. $10^{7}$ (ZMUS), Stavropol Territory, Stavropol City, Russky Forest, 9.III.2014, leg. R.V. Zuev.

REMARKS. Originally described from Central Asia [Attems, 1904], it is known also from Moldavia, eastern Ukraine, Crimea, Rostov-on-Don Region, Voronezh Region and Krasnodar Territory [Folkmanová, 1956; Titova, 1973]. Folkmanová [1956] described the subspecies E. retusidens ornatus Folkmanova, 1956, from the Krasnodar Territory and Rostov Region, which was later elevated to the rank of a species [Pereira, Hoffman, 1993]. E. ornatus differs from E. retusidens in the absence of prelabral non-areolate fields, as well as by lots of teeth and a small notch on the labrum. The specimen found in Stavropol definitely belongs to $E$. retusidens, although it has 57 pairs of legs, i.e. slightly higher than the maximum (55) specified by Titova [1973] for this species.

New illustrations are provided to document the identity (Figs 23-25).

\section{Schendyla nemorensis (C.L. Koch, 1837)}

Figs 26-30, Map 5.

MATERIAL. $1+$ (ZMUS), Stavropol Territory, Stavropol City, Tamansky Forest, 10.IX.2013; 1 (ZMUS), same locality, 1.IV.2014; 3 우 (ZMUS), same locality, 8.IV.2014; 1 (ZMUS), same locality, 17.V.2014; 1 q (ZMUM), same locality, 1.VI.2014; 1 오 (ZMUS), Mamaysky Forest, 6.II.2013; 1 으 (ZMUM), same locality, 16.III.2014; 1 q (ZMUS), Botanical Garden, 26.III.2014; 9 of (ZMUM), same locality, 10.IV.2014; 1 9 (ZMUS), same locality, 10.IV.2014; 5 우 (ZMUM), same locality, Picea plantation, 6.III.2014; 6 juv. (ZMUS), Shpakovsky Distr., near Grushevy, steppe, soil, 23.III.2014; 1 juv. (ZMUS), same locality, 14.VI.2014;

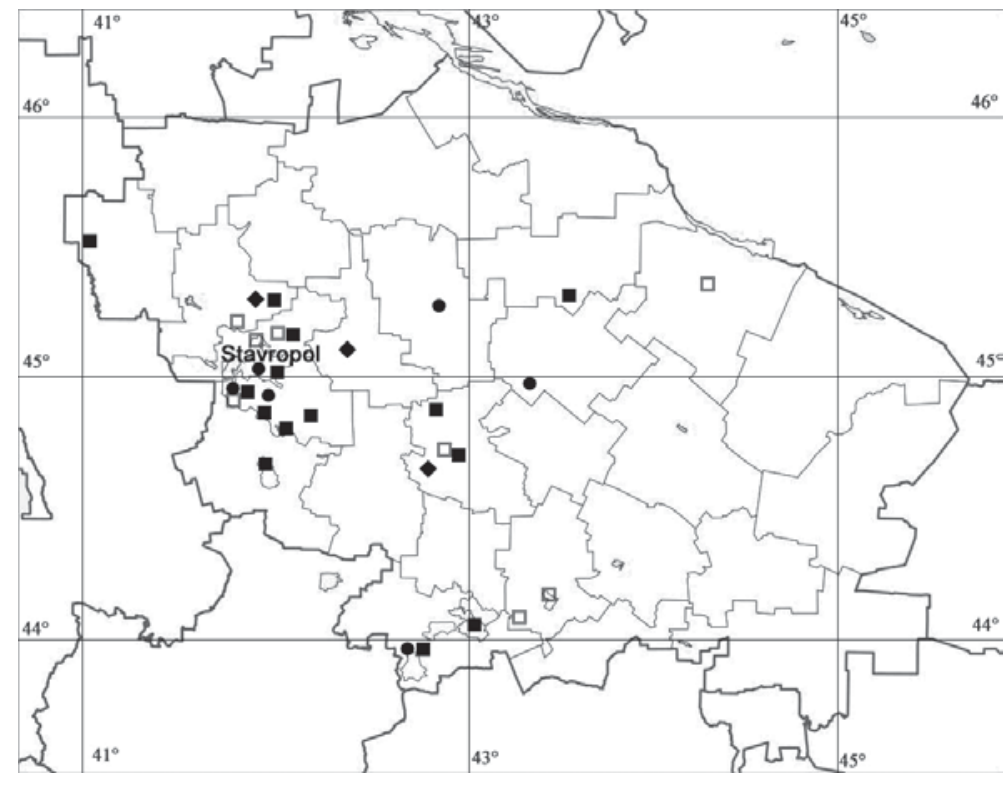

Map 5. Distribution of centipedes in the Stavropol Territory: circle - Schendyla nemorensis; square - Clinopodes caucasicus; diamond - Diphyonyx conjungens; open square Pachymerium ferrugineum.

Карта 5. Распространение губоногих многоножек в Ставропольском крае: круг Schendyla nemorensis; квадрат - Clinopodes caucasicus; ромб - Diphyonyx conjungens; незакрашенный квадрат - Pachymerium ferrugineum. 

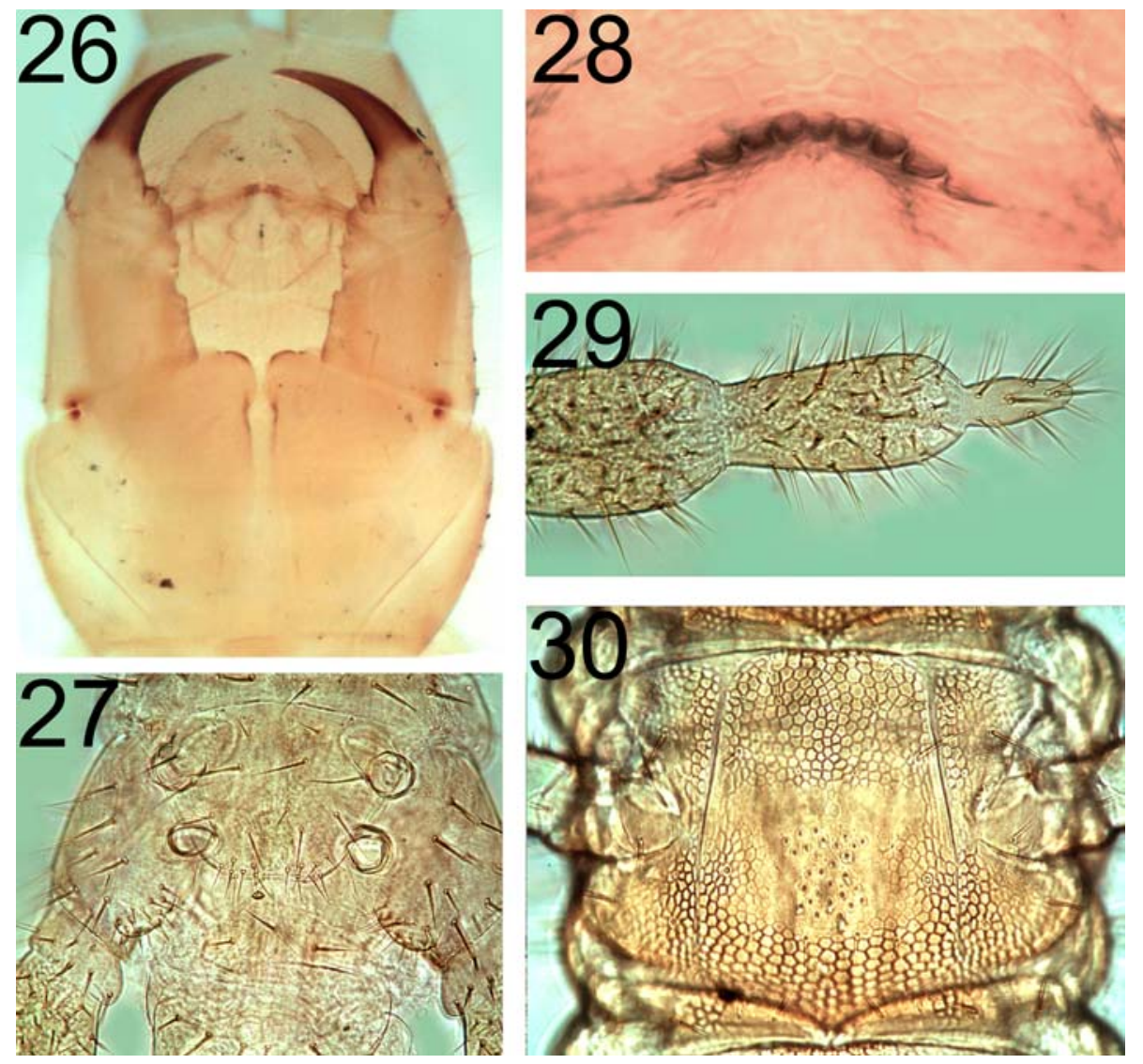

Figs 26-30. Schendyla nemorensis (C.L. Koch, 1837). 26 - forcipular segment, ventral view; 27 - terminal part of body, ventral view; 28 - labrum; 29 - tarsus of ultimate leg; 30 - intermediate part of trunk, ventral view. Pictures taken not to scale.

Pис. 26-30. Schendyla nemorensis (C.L. Koch, 1837). 26 - ногочелюсть, снизу; 27 - задний конец тела, снизу; 28 - лабрум; 29 - тарзус конечных ног; 30 - средняя часть тела, снизу. Фотографии без масштаба.

6 우, 10 juv. (ZMUS), near Tatarka, steppe, soil, 27.V.2013; 1 ㅇ (ZMUS), Petrovsky Distr., near Lake Solyonoe, steppe, 10.VII.2014 2 우, 2 juv. (ZMUS), Blagodarnensky Distr., near Elizavetinskoe, forest belt, 19.VI.2013; 1 juv. (ZMUS), Predgornyi Distr., near Kislovodsk, Borgustansky Ridge, forest, 20.VII.2013, all leg. R.V. Zuev.

REMARKS. A European species widespread all over Europe, it is also known from North Africa and introduced to North America [Simaiakis et al., 2013]. This is the first formal record of $S$. nemorensis in the Caucasus. In Russia, it has been reported from the Moscow, Kursk, Belgorod and Rostov-on-Don regions [Zalesskaja et al., 1982].

New illustrations are provided to document the identity (Figs 26-30).

\section{Family GEOPHILIDAE}

Clinopodes caucasicus (Sseliwanoff, 1883) Figs 31-33, Map 5.

MATERIAL. 12 우, 4 juv. (ZMUS), Stavropol Territory, Stavropol City, Tamansky Forest, soil, 27.VII.2010; 4 90, 4 juv. (ZMUS), same locality, 8.IX.2012; $1 \overbrace{}^{\prime}, 1$ (ZMUS), same locality, 13.X.2012; $2 \sigma^{7} \sigma^{7}, 2$ 우 (ZMUS), same locality, 14.IV.2013; 2 $\sigma^{7} \sigma^{7}, 4$ 우 (ZMUM), same locality, 28.IV.2013; 1 ᄋ', 2 우 (ZMUS), same locality, 15.VI.2013; 1 O', 1 + (ZMUS), same locality,
10.IX.2013; 3 우 (ZMUS), same locality, 12.IX.2013; $1 \sigma^{\top}, 1$ ㅇ, 1 juv. (ZMUS), same locality, 5.X.2013; 2 우 (ZMUS), same locality, 9.X.2013; $1 \sigma^{7}, 1$ (ZMUS), same locality, 11.I.2014; 2 우, 2 juv. (ZMUS), same locality, 18.I.2014; $6 \sigma^{7} \sigma^{7}, 10$ 우, 6 juv. (ZMUS), same locality, 15.II.2014; 1 ㅇ, 2 juv. (ZMUS), same locality, 8.IV.2014; $6 \sigma^{\top} \sigma^{\top}, 6$ 우, 11 juv. (ZMUS), same locality, 1.VI.2014; 3 O०, 3 juv. (ZMUM), Mamaysky Forest, 19.V.2010; 1 o (ZMUS), same locality, 27.X.2012; $2 \sigma^{7} \sigma^{7}, 2$ 우, 2 juv. (ZMUM), same locality, 31.III.2013; 2 + (ZMUS), same locality, 16.IV.2013; 1 ( (ZMUS), same locality, 29.VI.2013; 2 우 (ZMUS), same locality, 3.VIII.2013; $1 \sigma^{7}, 1$ ㅇ (ZMUS), same locality, 22.IX.2013; $3 \bigcirc^{7} \sigma^{7}, 3$ 우, 6 juv. (ZMUS), same locality, 16.III.2014; $1 \sigma^{\top}, 3$ 우 (ZMUS), Tatarsky Forest, 3.V.2013; 1 フ , 6 우 (ZMUM), same locality, 19.X.2013; 1 + (ZMUS), Russky Forest, 23.III.2013; 1 ○", 2 ㅇ, 6 juv. (ZMUS), same locality, 6.IV.2013; 1 juv. (ZMUS), same locality, 6.VII.2013; 2 juv. (ZMUS), same locality, 27.VII.2013; 1 juv. (ZMUS), same locality, 12.X.2013; $1 \sigma^{7}, 1$ ㅇ (ZMUS), same locality, 3.XI.2013; $2 \sigma^{7} \sigma^{7}, 1$ (ZMUS), same locality, 2.III.2014; 1 ○', 4 우, 4 juv. (ZMUS), same locality, 9.III.2014; 8 O $^{\top}$ ', 11 क, 5 juv. (ZMUM), same locality, 4.V.2014; $8 \sigma^{\top} \sigma^{\top}, 19$ 우 13 juv. (ZMUS), same locality, 10.V.2014; $8 \sigma^{7} \sigma^{\top}, 7$ 오 10 juv. (ZMUS), same locality, 7.VI.2014; $1 \bigcirc^{\top}, 1$ (ZMUS), same locality, 31.I.2015; $3 \sigma^{\top} \sigma^{\top}, 3$ 우, 2 juv. (ZMUS), Botanical Garden, 6.III.2014; 3 ○ $^{7}, 6$ 우, 6 juv. (ZMUS), same locality, 26.III.2014; 1 juv. (ZMUS), Shpakovsky Distr., near Grushevy, steppe, 28.X.2013; 2 ऽ $^{7}$ (ZMUS), same locality, 4.X.2014; 5 juv. (ZMUS), near Tsimlyansky, forest belt, 20.V.2013; 3 ठ $^{\top}, 2$ ㅇ, 6 juv. (ZMUS), near Rynok, Nedremannaya Mountain, forest, soil, 19.IV.2013; 2 9+, 1 juv. (ZMUS), same locality, 15.VII.2014; 1 ऽ, 2 (Z) (ZMUS), $5 \mathrm{~km}$ SSE of Izveshchatelnyi, Strizhament Mountain, forest, 3.VI.2010; $2 \sigma^{7} \sigma^{7}, 4$ 4 , 4 juv. (ZMUS), same locality, 

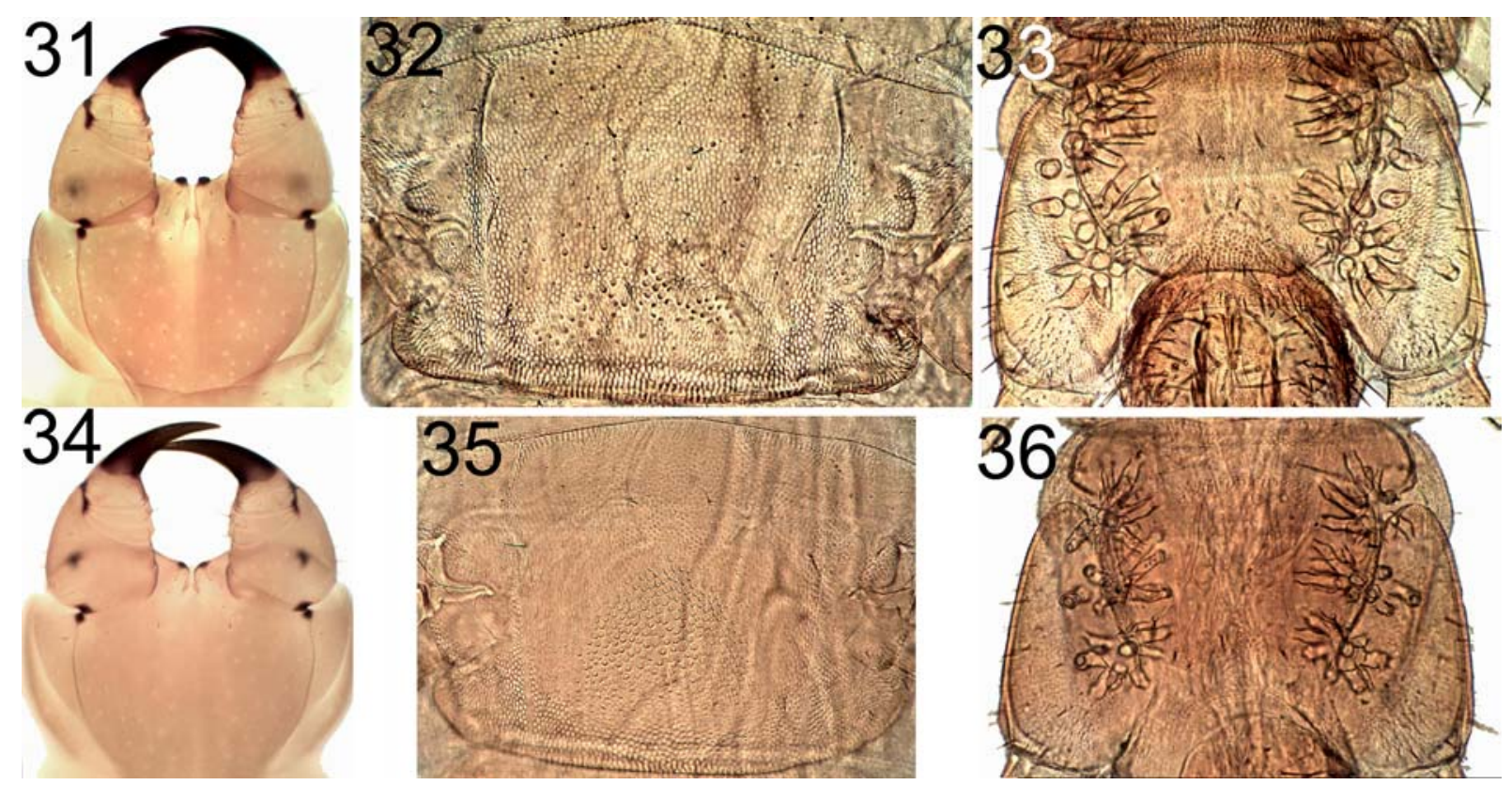

Figs 31-36. Clinopodes caucasicus (Sseliwanoff, 1883) (31-33) and Clinopodes flavidus C.L. Koch, 1847 (34-36). 31, 34 forcipular segment, ventral view; 32, 35 — posterior leg-bearing sternite; 33, 36 - ultimate leg-bearing segment, ventral view. Pictures taken not to scale.

Рис. 31-36. Clinopodes caucasicus (Sseliwanoff, 1883) (31-33) и Clinopodes flavidus Косh, 1847 (34-36). 31, 34 - ногочелюсть, снизу; 32, 35 - задний стернит; 33, 36 - сегмент конечных ног, снизу. Фотографии без масштаба.

9.VII.2014; $10^{7}, 1$ 오, 3 juv. (ZMUS), same locality, 11.VII.2014; 2 $\sigma^{7} \sigma^{\top}, 7$ 우, 1 juv. (ZMUM), Kochubeevsky Distr., Nevinnomyssk, confluence of Bolshoy Zelenchuk and Kuban rivers, floodplain forest, 7.IX.2014; 1 , 1 juv. (ZMUS), Alexandrovsky Distr., $7 \mathrm{~km}$ WNW of Alexandrovskoe, forest belt, 26.IV.2014; $1 \sigma^{7}, 1$,, 10 juv. (ZMUS), near Severnoe, forest belt, 26.IV.2014; $1 \mathrm{O}^{\text {T}}, 1$ \% (ZMUM), same locality, 26.IV.2014; 1 (ZMUS), Predgornyi Distr., near Pyatigorsk, Beshtau Mountain, forest, 21.VII.2013; 1 9 (ZMUS), near Kislovodsk, Borgustansky Ridge, forest, 20.VII.2013, all leg. R.V. Zuev; 1 \& (ZMUS), Turkmensky Distr., near Kulikovy Kopani, 25.VI.2014, leg. K.S. Priymak; 1 juv. (ZMUS), Shpakovsky Distr., Mikhaylovsk, Stavropol Scientific Research Institute of Agriculture, forest belt, 17.V.2006; 2 ㅇ (ZMUS), same locality, 8.VI.2007; $1 \sigma^{7}$ (ZMUS), same locality, 5.IV.2008; 1 + (ZMUS), same locality, field, 4.IV.2008; $3 \sigma^{7} \sigma^{7}, 3$ juv. (ZMUS), Izobilnensky Distr., near Moskovskoe, forest, 11.VII.2008; 1 (ZMUS), Novoalexandrovsky Distr., near Temigbeksky, forest belt, 24.V.2007; 1 क (ZMUS), same locality, 19.IV.2008; 1 (ZMUS), same locality, field, 19.IV.2008, all leg. M.B. Patyuta.

REMARKS. A Caucasian species also known from eastern Anatolia [Bonato et al., 2011]. It was described from Georgia and Azerbaijan [Sseliwanoff, 1883], recorded from the Stavropol Territory from near Kislovodsk [Muralewicz, 1907]. This seems to be the most common member of the order Geophilomorpha in forest habitats of the region. Illustrations are provided to document the identity (Figs 31-33).

\section{Clinopodes flavidus C.L. Koch, 1847}

Figs 34-36.

MATERIAL. $2 \sigma^{\top} \sigma^{\top}, 1$ q, 2 juv. (ZMUM), Stavropol Territory, Shpakovsky Distr., near Grushevy, bank of Lake Kravtsovo, abandoned garden, 4.X.2014; $1 \sigma^{7}$ (ZMUS), same locality, 4.X.2014, all leg. R.V. Zuev; 1 , 1 juv. (ZMUS), Mikhaylovsk, Stavropol
Research Institute of Agriculture, arable field, 31.III.2008, leg. M.B. Patyuta.

REMARKS. A Turano-Euro-Mediterranean species which Sseliwanoff [1883] and Lignau [1903] reported also from the Caucasus. In the Stavropol Territory, it is known from Pyatigorsk [Muralewicz, 1927]. Bonato et al. [2011] consider that the published records of $C$. flavidus from the Caucasus actually concern C. caucasicus. This is consistent with our observations. Thus, C. caucasicus is found everywhere in the region, mainly in forest habitats, while $C$. flavidus in the study area is rare, being confined to anthropogenous landscapes.

Illustrations are provided to document the identity, including the ventral pore-field of the posterior legbearing segment and the coxopleuron of the ultimate leg-bearing segment without an isolated pore (Figs 34-36).

Diphyonyx conjungens (Verhoeff, 1898) Figs 37-40, Map 5.

MATERIAL. 1 (ZMUM), Stavropol Territory, Grachevsky Distr., between Nagornyi and Bazovyi, forest belt, 26.IV.2014; 1 $\mathrm{O}^{7}$ (ZMUM), Alexandrovsky Distr., near Kruglolesskoe, pasture, 26.IV.2014, all leg. R.V. Zuev; $1 \mathrm{O}^{3}$ (ZMUS), Izobilnensky Distr., near Moskovskoe, pasture, 11.VII.2008, leg. M.B. Patyuta.

OTHER MATERIAL. $1 \mathrm{O}^{7}$ (ZMUS), Republic of Adygea, Maykopsky Distr., near Dakhovskaya, forest, 23.VI.2014; 1 + (ZMUM), same locality, outer wood, 24.VI.2014, all leg. R.V. Zuev.

REMARKS. This is the first formal record of $D$. conjungens in the Caucasus. An eastern Mediterranean species known from Turkey, Greece and the Crimea [Attems, 1929; Bonato et al., 2008]. Two more mem- 

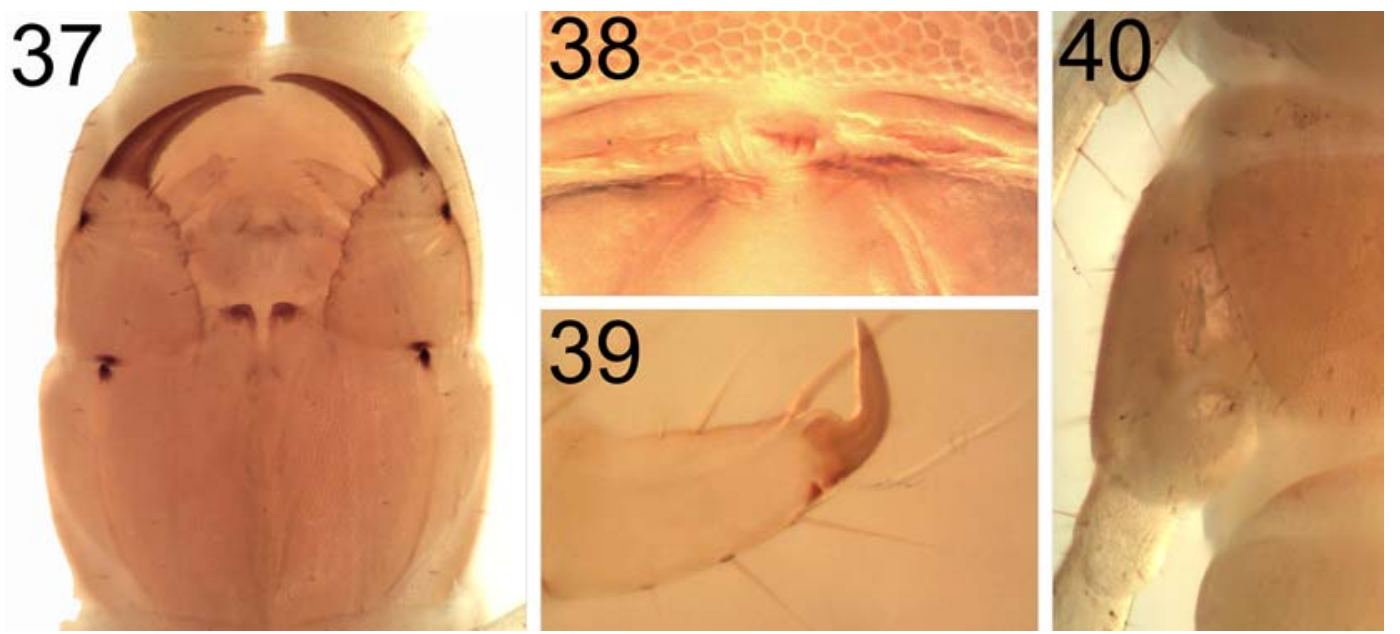

Figs 37-40. Diphyonyx conjungens (Verhoeff, 1898). 37 - forcipular segment, ventral view; 38 - labrum; 39 - distal part of right leg, anterior view; 40 - coxopleuron of ultimate leg-bearing segment, ventral view, taken not to scale.

Рис. 37-40. Diphyonyx conjungens (Verhoeff, 1898). 37 - ногочелюсть, снизу; 38 - лабрум; 39 - дистальная часть правой передней ноги, спереди; 40 - коксостернит правой конечной ноги, снизу. Фотографии без масштаба.

bers of this genus have been described from the Krasnodar Territory and Rostov Region: D. sukacevi (Folkmanova, 1956) and D. garutti (Folkmanova et Dobroruka, 1960); both these species differ from $D$. conjungens by the absence of teeth on the labrum [Folkmanová, 1956; Folkmanová, Dobroruka, 1960]. Thus, all three currently known species of the genus Diphyonyx inhabit the Caucasus.

New illustrations are provided to document the identity, including the characteristic fibulunguis on the anterior leg and two tubercles at the mid-point of the labrum (Figs 37-40).

\section{Geophilus flavus (De Geer, 1778) Map 3.}

MATERIAL. 1 (ZMUS), Stavropol Territory, Stavropol City, Tamansky Forest, 5.II.2013; 1 ( (ZMUS), same locality, 11.I.2014; 2 juv. (ZMUS), same locality, 8.IV.2014; 1 juv. (ZMUS), same locality, 17.V.2014; 1 +, 4 juv. (ZMUM), same locality, 1.VI.2014; 1 (ZMUM), Mamaysky Forest, 3.VIII.2013; 1 ㅇ (ZMUS), Botanical Garden, 26.III.2014; 1 q (ZMUS), same locality, Picea plantation, 26.III.2014; 2 juv. (ZMUS), Blagodarnensky Distr., near Elizavetinskoe, forest belt, 19.VI.2013, all leg. R.V. Zuev; 19 (ZMUS), Turkmensky Distr., near Kulikovy Kopani, 24.IV.2011, leg. Romaev.

REMARKS. A Euro-Siberian species, from the Caucasus it was recorded by Lignau [1903] from Krasnaya Polyana, Krasnodar Territory (referred to as G. longicornis austriacus Latzel, 1880).

\section{Pachymerium ferrugineum (Koch, 1835) Map 5.}

MATERIAL. 1 juv. (ZMUS), Stavropol Territory, Izobilnensky Distr., near Rogdestvenskaya, 18.V.2006; $3 \sigma^{7} \sigma^{7}, 1$ juv. (ZMUS), Shpakovsky Distr., Mikhaylovsk, Stavropol Scientific Research Institute of Agriculture, forest belt, 22.X.2006; 1 juv. (ZMUS), same locality, field, 4.IV.2008; 1 + (ZMUS), same locality, field, 5.IV.2008, all leg. M.B. Patyuta; 1 juv. (ZMUS), Stavropol City, Botanical Garden, 10.IV.2014; 2 juv. (ZMUS), Shpakovsky Distr., near Grushevyi, steppe, 23.V.2013; 2 juv. (ZMUS), same locality, 23.III.2014; 2 juv. (ZMUS), same locality, 14.VI.2014; 2 $\sigma^{7} \sigma^{7}, 1$, 6 juv. (ZMUM), same locality, 4.X.2014; 1 juv. (ZMUS), near Tatarka, steppe, 27.V.2013; 1 juv. (ZMUS), near Rynok, Nedremannaya Mountain, forest, soil, 19.IV.2013; 1 (ZMUS), same locality, steppe, 15.VII.2014; 1 juv. (ZMUS), Alexandrovsky Distr., $7 \mathrm{~km}$ WNW of Alexandrovskoe, forest belt, 26.IV.2014; 1 +, 1 juv. (ZMUS), Arzgirsky Distr., near Arzgir, steppe, in manure, 1.V.2014; 1 (ZMUM), same locality, 2.V.2015; 2 ㅇ, 30 juv. (ZMUS), Georgievsky Distr., Lysogorskaya, Lysaya Mountain, forest belt, soil, 19.VII.2013; 1 juv. (ZMUS), Georgievsk, Safonovsky Forest, 10.VIII.2013, all leg. R.V. Zuev; 2 O $^{7} \sigma^{7}, 2$ 우 (ZMUM), Georgievsk, farm, 9.VI.2014; 1 q (ZMUS), same locality, 10.VIII. 2014, all leg L.P. Ermolina.

REMARKS. A western Palaearctic species, in the Caucasus recorded from the Krasnodar Territory, Georgia and Azerbaijan [Lignau, 1903; Muralewicz, 1907, 1926]. In the Stavropol Territory, it inhabits mainly steppe biotopes, forest belts and fields, being very rare in the woods.

\section{Family LINOTAENIIDAE}

Strigamia aff. caucasia (Verhoeff, 1938)

Figs 41-44, Map 4.

MATERIAL. $1 \sigma^{\top}, 1$, 1 juv. (ZMUS), Stavropol Territory, Stavropol City, Mamaysky Forest, 27.X.2012; $1 \sigma^{\top}$ (ZMUS), same locality, 31.III.2013; 1 q (ZMUS), same locality, 28.IX.2013; 2 $\sigma^{\top} \sigma^{\top}, 2$ 20,1 juv. (ZMUS), same locality, 16.III.2014; $3 \sigma^{7}$ (ZMUS), same locality, 27.IX.2014; 1 juv. (ZMUS), Tamansky Forest, 13.X.2012; 1 juv. (ZMUS), same locality, 5.II.2013; 1 \% (ZMUS), same locality, 5.X.2013; $2 \sigma^{7} \sigma^{7}, 2$ 우 (ZMUS), same locality, 11.I.2014; 1 \% 1 juv. (ZMUS), same locality, 18.I.2014; 3 ○ $^{\top}, 7$ 우, 2 juv. (ZMUM), same locality, 15.II.2014; 1 juv. (ZMUS), same locality, 1.VI.2014; 1 ๆ (ZMUS), Russky Forest, 23.III.2013; $1 \sigma^{7}$ (ZMUS), same locality, 6.IV.2013; $1 \sigma^{7}$ (ZMUS), same locality, 12.X.2013; $2 \sigma^{7} \sigma^{7}, 1$ (ZMUM), same locality, 2.III.2014; 1 ○', 2 ㅇ (ZMUS), same locality, 9.III.2014; 1 ㅇ (ZMUS), same locality, 31.I.2015; $1 \sigma^{7}$ (ZMUS), Botanical Garden, 26.III.2014; 1 O' (ZMUS), same locality, 10.IV.2014; 1 o (ZMUS), Shpakovsky Distr., near Grushevyi, bank of Lake Kravts-

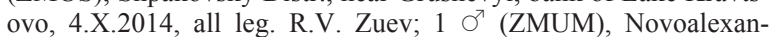
drovsky Distr., near Temigbeksky, forest belt, 19.IV.2008, leg. M.B. Patyuta. 

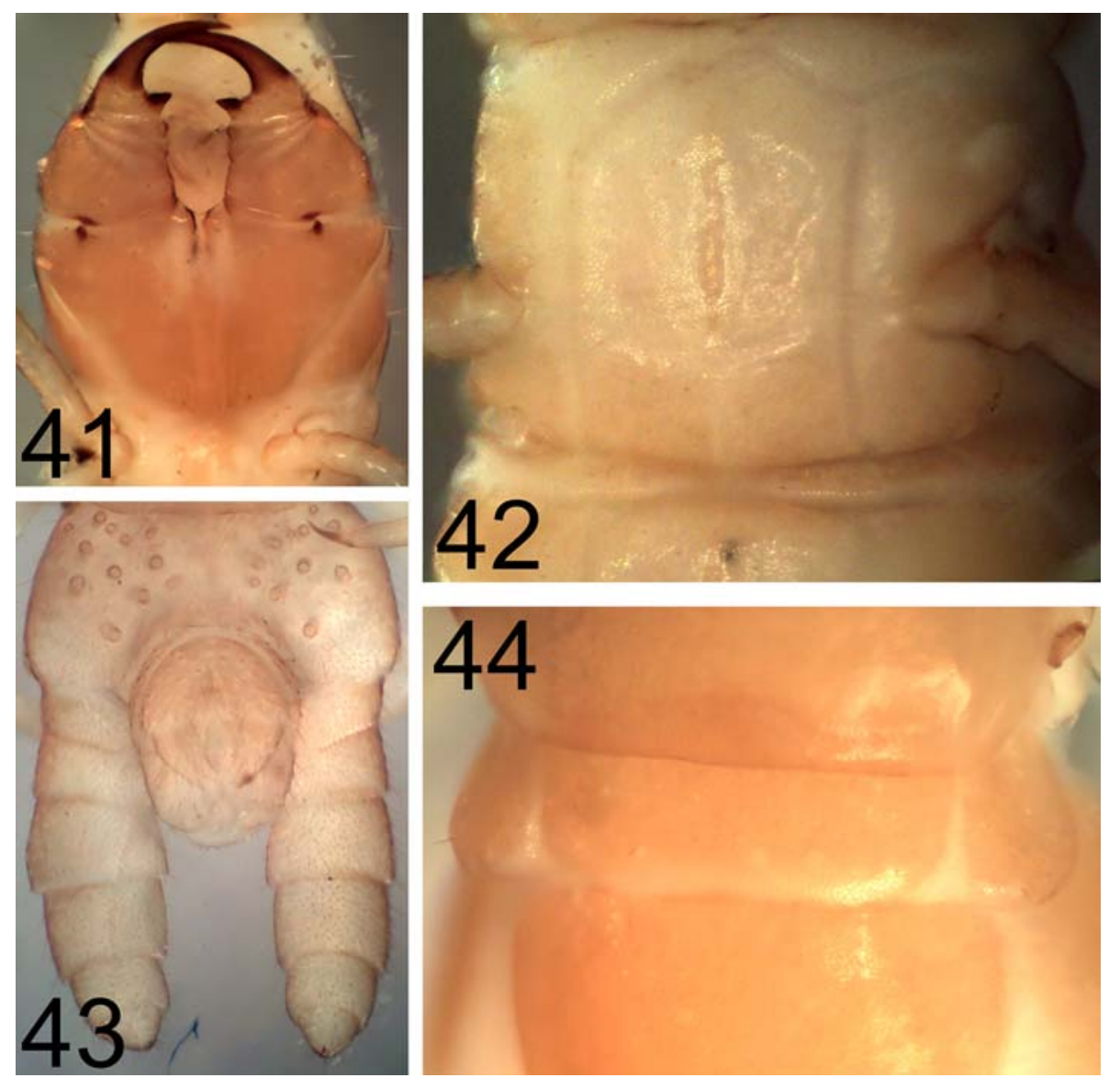

Figs 41-44. Strigamia aff. caucasia (Verhoeff, 1938). 41 - forcipular segment, ventral view; 42 - sternite of anterior leg-bearing segment; 43 - terminal part of body $\left(0^{7}\right)$, ventral view; 44 - ultimate pretergite. Pictures taken not to scale.

Рис. 41-44. Strigamia aff. caucasia (Verhoeff, 1938). 41 - ногочелюсть, снизу; 42 - передний стернит; 43 - задний конец тела $\left(0^{7}\right)$, снизу; 44 - претергит конечных ног. Фотографии без масштаба.

OTHER MATERIAL. $10^{\top}$ (ZMUS), Republic of Adygea, Maykopsky Distr., near Dakhovskaya, forest, leaf litter, 22.VI.2014; $\sigma^{7}$ (ZMUM), Krasnodar Territory, Apsheronsky Distr., Azish-Tau Ridge, near Cave Bol'shaya Azishskaya, $1500 \mathrm{~m}$ a.s.l., mixed forest, 2.VI.2012; $2 O^{7} O^{7}, 1$ +, 2 juv. (ZMUS), same locality, 18.VI.2014, all leg. R.V. Zuev.

REMARKS. A Caucasian species previously known only from a single locality in the western Caucasus, presumably the Abago Mountain [Verhoeff, 1938; Bonato et al., 2012]. The specimens we have found are similar to S. crassipes (C.L. Koch, 1835), a species already known from the Krasnodar Territory [Lignau, 1903], but they differ by the presence of sulci between the ultimate pretergite and intercalary pleurite on the ultimate leg-bearing segment (Figs 41-44).

\section{Conclusions}

As a result of our studies, the centipede fauna of the Stavropol Territory is found to include at least 28 species, 15 of which are recorded in the region for the first time. Three of them are new to the fauna of the Caucasus: Lithobius melanops, Diphyonyx conjungens and Schendyla nemorensis Species with the Caucasian distribution pattern (9) dominate the fauna of the re- gion. There are three species showing the European pattern; the Mediterranean, eastern Mediterranean and western Palaearctic chorotypes are represented by two species each. Each of the remaining patterns is only represented by a single member.

ACKNOWLEDGEMENTS. We thank all colleagues who rendered their material for the present study. We are also deeply grateful to Arkady A. Schileyko (Moscow, Russia), and G.Sh. Farzallieva (Perm, Russia) for the help in determining certain material to J.S. Volkova (Ulyanovsk, Russia) for carefully reviewing an advanced draft, and to S.I. Golovatch (Moscow, Russia) for providing much of the relevant literature and assisting in publication.

\section{References}

Attems C.G. 1904. Central- und hoch-asiatische Myriopoden. Gesammelt im Jahre 1900 von Dr. von Almassy und Dr. von Stummer // Zoologische Jahrbücher, Abteilung für Systematik, Ökologie und Geographie der Tiere. Bd.20. H.2. S.113-130.

Attems C.G. 1907. Myriopoden aus der Krim und dem Kaukasus, von Dr. A. Stuxberg gesammelt // Arkiv för Zoologi. Bd.3. No.25. S.1-16.

Attems C.G. 1929. Myriapoda. 1. Geophilomorpha // Das Tierreich. Lfg.52. S.1-388. 
Bonato L., Chagas Junior A., Dioguardi R., Edgecombe G., Lewis J., Minelli A., Pereira L., Shelley R., Stoev P., Uliana M. Zapparoli M. 2006. Chilobase - a world catalogue of Centipedes (Chilopoda). World Wide Web electronic publication. http://chilobase. bio.unipd.it [accessed 6-Jun-2015].

Bonato L., Zapparoli M., Minelli A. 2008. Morphology, taxonomy and distribution of Diphyonyx gen. n., a lineage of geophilid centipedes with unusually shaped claws (Chilopoda: Geophilidae) // European Journal of Entomology. Vol.105. P.343-354.

Bonato L., Iorio É., Minelli A. 2011. The centipede genus Clinopodes C.L. Koch, 1847 (Chilopoda, Geophilomorpha, Geophilidae): reassessment of species diversity and distribution, with a new species from the Maritime Alps (France) // Zoosystema. Vol.33. No.2. P.175-205.

Bonato L., Dányi L., Socci A.A., Minelli A. 2012. Species diversity of Strigamia Gray, 1843 (Chilopoda: Linotaeniidae): a preliminary synthesis // Zootaxa. Vol.3593. P.1-39.

Brandt J.F. 1840. Observations sur les espèces qui composent le genre Scolopendra suivies des caractères des espèces qui se trouvent dans le Muséum zoologique de l'Académie des Sciences de St.-Pétersbourg et de quelques coups d'oeil sur leur distribution géographique // Bulletin scientifique de l'Académie Imperiale des Sciences de Saint Pétersbourg. T.7. Fasc.11. P.148-160.

Eason E.H. 1972. The type specimens and identity of the species described in the genus Lithobius by C.L. Koch and L. Koch from 1841 to 1887 (Chilopoda: Lithobiomorpha) // Bulletin of the British Museum (Natural History), Zoology. Vol.22. P.105150 .

Eason E.H. 1997. On some Lithobiomorpha from the mountains of Kirghizia and Kazakhstan (Chilopoda) // Arthropoda Selecta. Vol.6. Nos 1-2. P.117-121.

Eichwald E. 1830. Zoologia specialis. Pars altera. Vilnae. IV, 233 pp.

Eichwald E. 1840-41. Fauna Caspio-Caucasiae nonnulis observationibus novis. illustr. cum. tab. lithogr. 40 // Nouvelles Mémoires de la Société Impériale des Naturalistes de Moscou. T.7 292 pp.

Folkmanová B. 1956. [On new forms of the order Geophilomorpha from the southern regions of the USSR. To the knowledge of centipedes of the USSR] // Zoologicheskii zhurnal. T.35. No.11. P.1633-1646 [in Russian].

Folkmanová B. 1958. [On new forms of Chilopoda from USSR)] // Zoologicheskii zhurnal T.37. No.2. P.183-192 [in Russian, with German summary].

Folkmanová B., Dobroruka L.J. 1960. [To the knowledge of centipedes (Chilopoda) of the USSR] // Zoologicheskii zhurnal. T.39. No.12. P.1811-1818 [in Russian, with German summary].

Kessler K.O. 1875. [On Russian Scolopendridae and Geophilidae] // Horae Societatis Entomologicae Rossicae. T.8. Fasc.1. P.2845 [in Russian].

Koch L. 1862. Die Myriapodengattung Lithobius. Nürnberg: J. L. Lotzbeck. $94 \mathrm{~S}$.

Lewis J.G.E. 2011. A review of the species in the genus Cryptops Leach, 1815 from the Old World related to Cryptops (Cryptops) hortensis (Donovan, 1810) (Chilopoda, Scolopendromorpha) // International Journal of Myriapodology. Vol.4. P.11-50.

Waldock J.M., Lewis J.G.E. 2014. Recent collections of centipedes from Christmas Island (Myriapoda: Chilopoda) // Raffles Bulletin of Zoology, Supplement No.30. P.71-80.

Lignau N.G. 1903. Die Myriopoden der Pontus-Küsten von Caucasus // Zapiski Novorossiyskago obshchestva estestvoispytateley. T.25. P.82-149 [in Russian and German].
Lignau N.G. 1915. Vielfüßer aus Abchasien // Ezhegodnik zoologicheskago muzeya Imperatorskoy Akademii nauk. T.18. P.349401 (for 1914).

Muralewicz W.S. 1907. Zur Myriapodenfauna des Kaukasus // Zoologischer Anzeiger. Bd.31. H.11-12. S.329-351.

Muralewicz W.S. 1926. Übersicht über die Chilopodenfauna des Kaukasus. II // Zoologischer Anzeiger. Bd.69. H.1-2. S.27-44.

Muralewicz W.S. 1927. [To the fauna of Myriapoda of the Caucasus] // Acta Societatis Entomologicae Stauropolitanae. T.3. No.1. P.4-7 [in Russian].

Muralewicz W.S. 1929. [Scutigeridae and Lithobiidae of the Caucasian fauna] // Mémoires de la Séction Zoologique de la Société des Amis des Sciences Naturelles, d'Anthropologie et d'Ethnographie. T.4. P.1-120 [in Russian].

Pereira L.A., Hoffman R.L. 1993. The American species of Escaryus, a genus of Holarctic centipeds (Geophilomorpha: Schendylidae) // Jeffersoniana. Vol.3. P.1-72.

Pocock R.I. 1888. On the Arachnida, Myriapoda and Crustacea of the Christmas Island // Proceedings of the Scientific Meetings of the Zoological Society of London. Year 1888. Pt.1. P. 556-564.

Reznik P.A. 1940. [Zoological notes I] // Trudy Voroshilovskogo pedagogicheskogo instituta. P. 193-198 [in Russian].

Simaiakis S.M., Zapparoli M., Minelli A., Bonato L. 2013. The centipede fauna (Chilopoda) of the island of Cyprus, with one new lithobiomorph species // Zootaxa. Vol.3647. No.2. P.279-306.

Sseliwanoff A.V. 1881. Myriapoden des Kaukasus // Trudy russkogo entomologicheskogo obshchestva, Peterburg. T.12. No.6. P.177-198 [in Russian].

Sseliwanoff A.V. 1883. [Materials towards the study of Russian myriapods] // Trudy russkogo entomologicheskogo obshchestva, Peterburg. T.18. P.69-121 [in Russian].

Titova L.P. 1973. [New species of the genus Escaryus Cook et Collins (Schendylidae Chilopoda)] // Ghilarov M.S. (ed.). Ekologiya pochvennykh bezpozvonochnykh. Moskva: Nauka. P.94-119 [in Russian].

Verhoeff K.W. 1938. Über einige Chilopoden des zoologischen Museums in München // Zoologischer Anzeiger. Bd.123. H.56. S.123-130.

Zalesskaja N.T. 1973. [The lithobiids (Chilopoda, Lithobiidae) of the Caucasus, their distribution and connection to the soil] // Ghilarov M.S. (ed.). Ekologiya pochvennykh bezpozvonochnykh. Moskva: Nauka. P.120-130 [in Russian].

Zalesskaja N.T. 1978. [Identification book of the lithobiomorph centipedes of the USSR (Chilopoda, Lithobiomorpha)]. Moskva: Nauka. 212 pp. [in Russian]

Zalesskaja N.T., Titova L.P., Golovatch S.I. 1982. [The myriapod fauna (Myriapoda) of the Moscow Area] // Ghilarov M.S. (ed.). Pochvennye bespozvonochnye Moskovskoy oblasti. Moskva: Nauka. P.179-200 [in Russian].

Zalesskaja N.T., Schileyko A.A. 1991. [Chilopods Scolopendromorphs (Chilopoda, Scolopendromorpha)]. Moskva: Nauka. P.1-102 [in Russian].

Zalesskaja N.T., Schileyko A.A. 1992. The distribution of Scolopendromorpha in the USSR (Chilopoda) // Meyer E., Thaler K., Schedl W. (eds). Advances in Myriapodology. Berichte des Naturwissenschaftlich-Medizinischen Vereins in Innsbruck, Supplement 10. S.367-372.

Zapparoli M. 1992. Note su tassonomia, corologia ed ecologia di Lithobius peregrinus Latzel, 1880 (Chilopoda: Lithobiomorpha) // Annalen des Naturhistorischen Museums in Wien, Serie B. Bd.93. S.161-179.

Responsible editor S.I. Golovatch 